\title{
C. elegans Mediator 15 permits low temperature-induced longevity via regulation of lipid and protein homeostasis
}

Dongyeop Lee ${ }^{1}$, Seon Woo A. An ${ }^{1}$, Yoonji Jung ${ }^{1}$, Yasuyo Yamaoka ${ }^{1}$, Youngjae Ryu ${ }^{4}$, Grace Ying Shyen $\mathrm{Goh}^{5}$, Arshia Beigi ${ }^{5}$, Dengke $\mathrm{Ma}^{6}$, Chang Man $\mathrm{Ha}^{4}$, Stefan Taubert ${ }^{5}$, Youngsook Lee ${ }^{1,3}$ and Seung-Jae V. Lee $e^{1,2, *}$

${ }^{1}$ Department of Life Sciences, ${ }^{2}$ School of Interdisciplinary Bioscience and Bioengineering, and

${ }^{3}$ Department of Integrative Bioscience \& Biotechnology, Pohang University of Science and Technology, Pohang, Gyeongbuk, South Korea, 37673

${ }^{4}$ Research Division, Korea Brain Research Institute, Daegu, South Korea, 41068

${ }^{5}$ Centre for Molecular Medicine and Therapeutics, BC Children's Hospital Research Institute, Department of Medical Genetics, University of British Columbia, 950 W 28th Ave, Vancouver, BC V5Z 4H4, Canada

${ }^{6}$ Cardiovascular Research Institute and Department of Physiology, University of California San Francisco, San Francisco, CA, USA, 94158

*Correspondence: seungjaelee@ postech.ac.kr 


\begin{abstract}
Low temperatures slow aging and extend lifespan in many organisms, including Caenorhabditis elegans. However, the metabolic and homeostatic aspects of low temperature-induced longevity remain poorly understood. Here, we show that changes in lipid composition regulated by MDT15/Mediator 15, transcriptional co-regulator, is essential for low temperature-induced longevity and proteostasis in C. elegans. We find that inhibition of $m d t-15$ prevents animals from living long at low temperatures. We show that MDT-15 up-regulates fat-7, a fatty acid desaturase, at low temperatures, which increases the ratio of unsaturated to saturated fatty acids. We further demonstrate that maintaining this increased fatty acid ratio is essential for protein homeostasis and longevity at low temperatures. Thus, the homeostasis of lipid composition by MDT-15 appears to be a limiting factor for C. elegans proteostasis and longevity at low temperatures. Our findings highlight the crucial roles of fat regulation in maintaining normal organismal physiology under different environmental conditions.
\end{abstract}

\title{
Introduction
}

Environmental temperature has a major influence on organismal physiology, including growth, metabolism, and aging. The body temperature of poikilothermic organisms, such as Caenorhabditis elegans, is subject to changes in environmental temperatures; these organisms live long at low ambient temperatures but short at high temperatures (Conti, 2008; Jeong et al., 2012). In addition, mice with reduced body temperature live long (Conti et al., 2006), suggesting potentially conserved mechanisms across diverse species. Several genetic factors have been 
identified to modulate temperature-dependent lifespan changes in C. elegans (Chen et al., 2016; Horikawa et al., 2015; Lee and Kenyon, 2009; Xiao et al., 2013). However, the metabolic processes underlying this phenomenon remain poorly understood.

The relative proportion of unsaturated fatty acids (UFAs) and saturated fatty acids (SFAs) is homeostatically regulated in various organisms (Holthuis and Menon, 2014). In C. elegans, the UFA/SFA ratio is inversely proportional to the environmental temperature (Tanaka et al., 1996). This is consistent with the idea that more UFAs than SFAs are required for the maintenance of physical properties of lipids in biological membranes at low temperature (Holthuis and Menon, 2014). Changes in the UFA/SFA ratio is also important for C. elegans to adjust its growth to high or low temperatures (Ma et al., 2015; Svensk et al., 2013). (Taubert et al., 2006; Van Gilst et al., 2005)However, the mechanisms by which the UFA/SFA ratio regulates the lifespan of organisms at different environmental temperatures remain largely unknown.

Here, we showed that MDT-15/Mediator 15, a subunit of the Mediator complex for RNA polymerase II-regulated transcription, has differential effects on lifespan at different environmental temperatures. We found that $m d t-15$ mutations prevented worms from living long at low temperatures. We showed that $m d t-15$ was required for expressing fat-7, a fatty acid desaturase crucial for increasing the UFA/SFA ratios at low temperatures. Furthermore, we demonstrated that a homeostatic increase in the UFA/SFA ratios at low temperatures was critical for longevity and chaperone-mediated proteostasis. These data suggest that MDT-15 is a limiting factor in the low temperature-mediated longevity of $C$. elegans, and it exerts this effect via the maintenance of the physical properties of lipids and protein homeostasis. 


\section{Results}

\section{mdt-15 is required for longevity and organismal fitness at low temperatures}

MDT-15/Mediator 15 is a large subunit of the Mediator complex that regulates diverse physiological processes, including fat metabolism, stress resistance, and lifespan (Allen and Taatjes, 2015). While performing experiments for our previous reports (Artan et al., 2016; Lee et al., 2015), we found that reduction of function $m d t-15$ mutations greatly suppressed the long lifespan of $C$. elegans at a low temperature $\left(15^{\circ} \mathrm{C}\right)$ (Fig. 1A; Supplementary Fig. S1A). In contrast, $m d t-15(-)$ mutations had a marginal effect on the lifespan at a high temperature $\left(25^{\circ} \mathrm{C}\right)$ (Fig. 1A; Supplementary Fig. S1A). To further confirm this result, we employed an auxininducible degron system using CRISPR/Cas9 knock in (Zhang et al., 2015), and generated $m d t$ 15::degron::EmGFP strains (Fig. 1B). We found that auxin-induced depletion of MDT-15 (Fig. 1C; Supplementary Fig. S1B, C) substantially suppressed longevity at $15^{\circ} \mathrm{C}$ (Fig. 1D; Supplementary Fig. S1D). Next, we examined the effects of an $m d t-15$ gain of function (gof) mutation (Svensk et al., 2013), which we generated in a wild-type background by using CRISPR, on lifespan at different temperatures. We found that the $m d t-15(g o f)$ mutations did not affect the lifespan at $25^{\circ} \mathrm{C}$ or $15^{\circ} \mathrm{C}$ (Fig. 1E). Together, these data indicate that MDT-15 is required but not sufficient for increasing the adult lifespan at low temperatures.

Next, we determined whether $m d t-15$ is important for maintaining the overall fitness, including the growth and reproduction of $C$. elegans at different temperatures. $m d t-15(-)$ mutants exhibited a greater impact on body size at low temperatures than at high temperatures (Fig. 1F; Supplementary Fig. S1E). In addition, the defects in reproduction caused by $m d t-15(-)$ mutations were more pronounced at a low temperature $\left(15^{\circ} \mathrm{C}\right)$ than at a high temperature $\left(25^{\circ} \mathrm{C}\right)$ 
(Fig. 1G, H; Supplementary Fig. S1F). Together, these data suggest that MDT-15 plays a role in longevity and fitness more substantially at low temperatures than at high temperatures.

\section{MDT-15 regulates the proper expression of fatty acid desaturases at low temperatures}

Next, we sought to identify genes whose expression was affected by MDT-15 at different temperatures. Our RNA seq. analysis indicates that 79 genes were up-regulated and 253 genes were down-regulated at $15^{\circ} \mathrm{C}$ in an MDT-15-dependent manner (fold change $>1.5$ and $p$ values $<0.05$; Fig. 2A-C; Supplementary Fig. S2A-F). Gene ontology (GO) analysis showed that GO terms involved in diverse metabolic processes, including aminoglycan, fatty acid, and carboxylic acid metabolism, were enriched in MDT-15-dependent up-regulated genes at $15^{\circ} \mathrm{C}$ (Fig. 2D). GO terms, including chromatin assembly, aminoglycan catabolic process, response to heat, immune system process, and response to unfolded protein, were overrepresented among the MDT-15dependent down-regulated genes at $15^{\circ} \mathrm{C}$ (Fig. 2E).

Based on our GO analysis showing "metabolism" terms enriched among genes upregulated at $15^{\circ} \mathrm{C}$ in an MDT-15-dependent manner, we further analyzed our RNA seq. data with a focus on genes located in carbohydrate and lipid metabolic pathways (Fig. 3A). We found that genes that directly affect lipid metabolism, including fatty acid synthesis, lipolysis, lipid transport, and fatty acid $\beta$-oxidation, tended to be more up- or down-regulated by changes in temperature and $m d t-15(-)$ mutation than those involved in glycolysis and the Krebs cycle (Fig. 3A). In addition, the expression of many fatty acid desaturases and elongases involved in fatty acid biosynthesis was down-regulated in $m d t-15(-)$ mutants, except elo-8 (Fig. 3A). In contrast, the expression of genes involved in lipolysis, lipid transport, and fatty acid $\beta$-oxidation did not 
show a consistent pattern at different temperatures or by $m d t-15(-)$ mutations (Fig. 3A). These data suggest that MDT-15 up-regulates fatty acid biosynthesis and desaturation at low temperatures.

We noticed that the expression of fat-7 was the most strongly associated with temperature and MDT-15 genetic background changes (Fig. 3A, B). Among all seven fatty acid desaturases, the expression level of fat-7 was significantly higher at $15^{\circ} \mathrm{C}$ than at $25^{\circ} \mathrm{C}$ (Ma et al., 2015), and this was MDT-15 dependent (Fig. 3B). In contrast, the other six fatty acid desaturase genes did not display MDT-15-dependent changes in the expression at different temperatures (Fig. 3B). qRT-PCR data for fat-2, fat-5, fat-6, and fat-7 mRNAs and fat-7::GFP transgenic animals were generally consistent with our RNA seq. data (Fig. 3C, D; Supplementary Fig. S2GJ). Together, these data suggest that MDT-15 specifically increases the expression of fat-7 at low temperatures.

\section{mdt-15 mutations decrease the UFA/SFA ratio}

Fatty acid desaturases and MDT-15 are crucial for de novo fat synthesis (Grants et al., 2015). By using Oil red O staining, we showed that the overall fat levels were not changed at $15^{\circ} \mathrm{C}$ in wildtype animals, but were greatly reduced in $m d t-15(-)$ mutants at $15^{\circ} \mathrm{C}$ (Fig. $\left.4 \mathrm{~A}, \mathrm{~B}\right)$. At $15^{\circ} \mathrm{C}, m d t$ 15(-) mutants also displayed a pale intestine phenotype (Fig. 4C), which correlates with low fat levels (McKay et al., 2003). These data suggest that MDT-15 is crucial for maintaining overall fat levels at low temperatures.

We then examined the effects of low temperature and $m d t-15(-)$ mutations on the relative proportion of SFAs and UFAs because fatty acid desaturases increase the unsaturated 
status of fatty acids. The levels of SFAs, including C14:0 and C16:0, were reduced at $15^{\circ} \mathrm{C}$ (Fig. 4D); this was consistent with the findings of a previous report (Tanaka et al., 1996). In contrast, the levels of several UFAs, including C16:1n-7, C18:1n-7, and C20:4n-3, were increased at $15^{\circ} \mathrm{C}$ (Fig. 4D). The overall levels of SFAs were decreased, whereas those of UFAs were increased in the wild-type at $15^{\circ} \mathrm{C}$ (Fig. 4E, F), and this led to significant increases in the UFA/SFA ratio (Fig. 4G). Notably, mdt-15(-) mutations reduced the effect of low temperature on changes in the SFA and UFA levels (Fig. 4E, F). This resulted in a reduced UFA/SFA ratio in $m d t-15(-)$ mutants, which was more pronounced at $15^{\circ} \mathrm{C}$ than at $25^{\circ} \mathrm{C}$ (Fig. 4G). These data suggest that MDT-15 is crucial for increasing the UFA/SFA ratio at low temperatures.

\section{Low UFA/SFA ratio suppresses longevity at low temperatures}

Having established that $m d t-15$ is crucial for maintaining overall fat levels and increasing the UFA/SFA ratio at low temperature, we sought to determine which of these two effects was critical for low temperature-induced longevity. Several lines of evidence based on genetic and dietary interventions suggest that decreasing the UFA/SFA ratio results in a short lifespan at low temperatures rather than a reduction in overall fat levels. First, fat-6(-); fat-7(-) double mutations, which increase SFA levels and decrease overall fat levels (Brock et al., 2007), significantly and specifically shortened lifespan at $15^{\circ} \mathrm{C}$ but not at $25^{\circ} \mathrm{C}$ (Fig. 5A). Second, mutations in the paqr-2/adiponectin receptor, which decrease the expression of fat-7 and increase the levels of SFA (Svensk et al., 2013), largely suppressed longevity at $15^{\circ} \mathrm{C}$ (Fig. 5B). Third, nhr-49 mutations, which decreased fat-7 mRNA levels and the UFA/SFA ratio while increasing overall fat levels (Van Gilst et al., 2005) (Supplementary Fig. S3A-C), significantly shortened 
longevity at $15^{\circ} \mathrm{C}$ (Fig. 5C). The lifespan of $n h r-49 ; m d t-15$ double mutants at low temperature was similar to that of the single mutants (Fig. 5D), suggesting that MDT-15 and NHR-49 act together to increase the longevity at low temperature. Fourth, glucose-enriched diets, which decrease the UFA/SFA ratio while increasing the overall fat levels (Devkota et al., 2017; Lee et al., 2015; Pang et al., 2014), also substantially shortened longevity at $15^{\circ} \mathrm{C}$ (Fig. 5E, F) but not at $25^{\circ} \mathrm{C}$ (Fig. 5E, F) (Brokate-Llanos et al., 2014; Lee et al., 2015). Taken together, these data suggest that increasing the UFA/SFA ratio, rather than maintaining the overall fat levels, is essential for the longevity of C. elegans at low temperatures.

\section{Disruption of MDT-15-mediated fatty acid desaturation decreases proteostasis at low temperature}

How does fatty acid composition affect longevity at low temperatures? In our RNA seq. analysis, we noticed that the GO terms "response to heat" and "response to unfolded protein" were enriched among genes down-regulated by low temperature in an MDT-15-dependent manner (Fig. 2E). We investigated the functional significance of the enrichment of these GO terms by focusing on our research at low temperature because protein folding and homeostasis are crucial for healthy aging and longevity (Higuchi-Sanabria et al., 2018). We found that reduced expression of cytosolic chaperones, such as hsp-16.1, hsp-16.11, hsp-16.49, hsp-16.48, hsp-16.41, hsp-16.2, F44E5.4 (Hsp70), and F44E5.5 (Hsp70), at $15^{\circ} \mathrm{C}$ was highly elevated in $m d t-15(-)$ mutants (Fig. 6A). We confirmed this by measuring $h s p-16.1 / 11$ and F44E5.4/5 mRNA levels using $h s p-16.1:: G F P$ reporter and qRT-PCR (Fig. 6B-D). In contrast to the cytosolic chaperones, low temperatures or $m d t-15(-)$ mutations had a small or no effect on the expression of 
endoplasmic reticulum or mitochondrial chaperones (Fig. 6E-G). Additionally, paqr-2(-) mutations induced the expression of $h s p-16.1 / 11$ at $15^{\circ} \mathrm{C}$ (Supplementary Fig. S4). These data suggest that a reduction in the UFA/SFA ratio induces the expression of cytosolic chaperones at low temperatures.

Next, we determined how the genetic inhibition of $m d t-15$ increased the expression of cytosolic chaperones at low temperatures. We hypothesized that the inhibition of $m d t-15$ at low temperature increased proteotoxicity, which in turn induced chaperone gene expression as an adaptive response. Consistent with our hypothesis, $m d t-15$ mutations significantly increased the number of Q40::YFP puncta (Fig. 6H, I), a poly-glutamine proteotoxicity model in C. elegans (Morley et al., 2002). HSF-1/heat shock factor 1 is a major transcription factor that regulates the expression of cytosolic chaperones (Higuchi-Sanabria et al., 2018); hsf-1 knockdown suppressed the induction of $h s p-16.1:: G F P$ by $m d t-15$ RNAi at $15^{\circ} \mathrm{C}$ (Fig. $\left.6 \mathrm{~J}\right)$. Importantly, $h s f-1$ knock down exacerbated Q40::YFP aggregation in mdt-15 RNAi-treated worms but did not significantly affect the number of the aggregates in control worms at $15^{\circ} \mathrm{C}$ (Fig. $\left.6 \mathrm{~K}, \mathrm{~L}\right)$. These data imply that HSF-1-mediated chaperone induction is an adaptive response to increased proteotoxicity caused by the inhibition of $m d t-15$ at $15^{\circ} \mathrm{C}$. Altogether, our data suggest that the homeostatic regulation of lipid composition regulated by MDT-15 is crucial for maintaining proteostasis and longevity at low temperatures.

\section{Discussion}

\section{UFA/SFA ratio limits longevity at low temperatures}


In this study, we showed that $m d t-15$ was required for the longevity of $C$. elegans at low temperatures. When worms were cultured at a low temperature $\left(15^{\circ} \mathrm{C}\right)$, MDT-15 increased the UFA/SFA ratio by permitting the expression of several fatty acid desaturases, including fat-7. This homeostatic regulation of lipid metabolism by MDT-15 was essential for the longevity of $C$. elegans at low temperatures. Consistent with this possibility, $m d t-15(-)$ mutations reduced the UFA/SFA ratio and suppressed the low temperature-induced longevity. Similar to $m d t-15(-)$ mutations, fat-6(-); fat-7(-), paqr-2(-), and $n h r-49(-)$ mutations and glucose-enriched diets suppressed longevity at low temperatures. The reduction in the UFA/SFA ratio at low temperatures led to proteotoxicity, resulting in shortened lifespan. Previous studies have demonstrated that modulating the UFA/SFA ratio is crucial for larval development or resistance against environmental stress caused by extreme fluctuations in temperature (Brock et al., 2007; Ma et al., 2015; Murray et al., 2007; Svensk et al., 2013). Our data suggest that the homeostatic regulation of the UFA/SFA ratio is essential for the maintenance of proteostasis and longevity in adult $C$. elegans at low temperatures.

\section{MDT-15 regulates lifespan in response to changes in temperature and diet}

C. elegans MDT-15 is a transcriptional co-regulator involved in the physiological response to ingested materials, including food, toxins, pathogens, and stressors (Goh et al., 2014; PukkilaWorley et al., 2014; Schleit et al., 2011; Taubert et al., 2008; Taubert et al., 2006; Yang et al., 2006). Previously, we showed that MDT-15-regulated lipid metabolism is crucial for maintaining normal lifespan in glucose-enriched nutrient conditions (Lee et al., 2015). In this study, we demonstrated that MDT-15 was important for longevity at low ambient temperatures. Together, 
these findings suggest that MDT-15 plays a key role in adapting to changes in environmental temperatures and to various ingested materials. How MDT-15 differentially incorporates various inputs to exert proper cellular and physiological responses currently remains elusive. Because MDT-15 is a subunit of the Mediator complex that interacts with diverse transcription factors (Allen and Taatjes, 2015), it is possible that MDT-15 binds to these transcription factors differently under different conditions.

\section{Some specific transcription factors acting with MDT-15 may regulate the temperature- dependent expression of fat-7}

Among the seven $C$. elegans fatty acid desaturases, the expression of fat-7 was down-regulated at high temperature, instead of being up-regulated at low temperature, in an MDT-dependent manner (Fig. 3B). This suggests that the activity of some transcription factors, including MDT15, NHR-49, SBP-1, DAF-16/FOXO, and HSF-1, that induce fat-7 (Brunquell et al., 2016; Murphy et al., 2003; Taubert et al., 2006; Yang et al., 2006) is reduced at high temperatures. The effect of temperature on the activity of these transcription factors has not been investigated, except for the heat-activated HSF-1. However, HSF-1 positively regulates the expression of fat-7 at high temperatures (Brunquell et al., 2016), and this is in contrast to the data shown in this study. A recent study has shown that acyl-CoA dehydrogenase, ACDH-11, whose expression is up-regulated at high temperatures, negatively regulates fat-7 via NHR-49 (Ma et al., 2015), which acts with MDT-15 for target gene expression (Taubert et al., 2006). Thus, NHR-49 with MDT-15 may regulate the temperature-dependent expression of fat-7. 


\section{MDT-15-regulated membrane fluidity may influence lifespan}

The UFA/SFA ratio is crucial for maintaining the membrane fluidity for adaptation to different temperatures (Holthuis and Menon, 2014). In C. elegans, the rigid membrane leads to developmental defects at low temperatures (Svensk et al., 2016) and hyper-fluidic membrane decreases resistance against high temperatures (Ma et al., 2015). Here, we showed that $m d t$ 15(-) mutations decreased the UFA/SFA ratio at low temperature and suppressed the longevity. We also found that genetic interventions and glucose-enriched diets, which decrease the UFA/SFA ratio, shortened lifespan specifically at low temperatures. Thus, a reduction in the UFA/SFA ratio at low temperatures may increase the rigidity of membranes to a harmful level, which in turn may shorten lifespan. A recent study has demonstrated that feeding worms with glucose or glycolysis metabolites increases their membrane rigidity by altering bacterial metabolism (Devkota et al., 2017). However, we showed that feeding C. elegans with a glucoseenriched diet along with dead bacteria also shortened lifespan substantially at low temperature (Fig. 5F). Therefore, glucose-rich diets appear to directly shorten the lifespan of C. elegans at low temperatures independently of bacterial metabolism.

\section{MDT-15 regulates proteostasis independently of mitochondrial stress}

Mitochondrial stress, or a mild disruption of mitochondrial functions, up-regulates the expression of cytosolic chaperones (Kim et al., 2016; Labbadia et al., 2017). Therefore, it is possible that the induction of cytosolic heat shock proteins in $m d t-15(-)$ mutants observed in this study implicates mitochondrial stress. However, several lines of evidence are against this possibility. First, we found that $m d t-15(-)$ mutations had a small or no effect on the induction of mitochondrial stress 
response genes. Second, unlike the increase in fat levels required for the induction of cytosolic chaperones under mitochondrial stress (Kim et al., 2016), we showed that $m d t-15(-)$ mutations reduced overall fat levels while increasing cytosolic chaperone levels. Third, different from mitochondrial perturbations that improve proteostasis (Kim et al., 2016; Labbadia et al., 2017), we found that the inhibition of $m d t-15$ disrupted proteostasis. Overall, our data suggest that the induction of cytosolic chaperones by $m d t-15(-)$ mutations is a response to increased proteotoxicity and is distinct from mitochondrial stress.

\section{Low UFA/SFA ratios are associated with various human diseases}

Recent reports indicate that supplementing with certain types of UFAs is sufficient to extend the lifespan of C. elegans (Han et al., 2017; O'Rourke et al., 2013). Together with our current report, these findings suggest the beneficial effects of UFAs on healthy aging. Interestingly, low UFA/SFA ratios are associated with many diseases such as Niemann-Pick disease, hypertension, heart disease, and Alzheimer's disease (Chi and Gupta, 1998; Soderberg et al., 1991; Sztolsztener et al., 2012; Wang et al., 2003). Human fibroblasts that carry mutations in NPC1, the gene responsible for the Niemann-Pick type C disease, display high levels of SFAs and a reduced membrane fluidity (Sztolsztener et al., 2012). Low UFA/SFA ratios also positively correlate with heart disease incidences in humans (Wang et al., 2003). Spontaneously hypertensive rats show lower UFA/SFA ratio in aorta and kidney than normotensive rats (Chi and Gupta, 1998). Patients of Alzheimer's disease show very high levels of SFAs in their brains (Soderberg et al., 1991), and this is consistent with our data that show the occurrence of proteotoxicity because of low UFA/SFA ratios. These findings point toward the importance of low UFA/SFA ratios in the 
pathophysiology of the disease. Further research on MDT-15 and lipid metabolism using $C$. elegans may provide useful information that may eventually help the treatment of human diseases because functions of the Mediator complex are evolutionarily well conserved (Allen and Taatjes, 2015).

\section{Homeostatic regulation of lipid metabolism is crucial for longevity}

Homeostatic regulation of proteins and DNA is important to prevent premature aging and promote longevity (Lopez-Otin et al., 2013). In addition, recent studies indicate that RNA quality control mediated by nonsense-mediated mRNA decay and accurate mRNA splicing contributes to the longevity in C. elegans (Heintz et al., 2017; Son and Lee, 2017; Son et al., 2017; Tabrez et al., 2017). Our current findings suggest that lipid homeostasis is also crucial for longevity at low temperatures, perhaps because lipids are very susceptible to changes in environmental temperatures. We further showed that the disruption of lipid composition at low temperatures increased proteotoxicity and shortened lifespan. Thus, the homeostatic regulation of lipids and proteins is most likely to be interconnected and tightly regulated for health and longevity.

\section{Materials and Methods}

Strains. All strains were maintained on nematode growth medium (NGM) agar plates seeded with $E$. coli (OP50) at $20^{\circ} \mathrm{C}$. Following strains were used in this study. N2 wild-type, IJ 235 mdt15(tm2182) III obtained by outcrossing XA7702 (a gift from Stefan Taubert lab) four times to Lee lab N2, IJ1648 ieSi57[eft-3p::TIR1::mRuby::unc-54 3'UTR; cb-unc-119] II outcrossed four 
times to Lee lab N2, IJ1651 yh44[mdt-15::degron::EmGFP] III outcrossed four times to Lee lab N2 after CRISPR/Cas9 editing, IJ1729 ieSi57[eft-3p::TIR1::mRuby::unc-54 3'UTR; cb-unc-119] II; yh44[mdt-15::degron::EmGFP] III obtained by crossing IJ1648 and IJ1651, IJ1468 mdt15(yh8) III outcrossed four times to Lee lab N2 after CRISPR/Cas9 editing, DMS303 nIs590[fat7p::fat-7::GFP] V. IJ1742 mdt-15(tm2 182) III; nIs590[fat-7p::fat-7::GFP] V obtained by crossing IJ235 and DMS303, IJ511 fat-6(tm331) IV; fat-7(wa36) V outcrossed seven times to Lee lab N2, IJ666 paqr-2(tm3410) III obtained by outcrossing QC121 (a gift from Marc Pilon lab) four times to Lee lab N2, CF2774 $n h r-49(g k 405) I$ outcrossed four times to N2, IJ360 $n h r$ 49(gk405) I; mdt-15(tm2182) III, obtained by crossing CF2774 and IJ235. IJ1625 skn-1(zj15) outcrossed four times to Lee lab N2. IJ414 Is[hsp-16.1p::hsp-16::gfp; rol-6D] (a gift from Junho Lee lab). IJ184 rmIs133[unc-54p::Q40::YFP] outcrossed four times to Lee lab N2. IJ1740 mdt15(tm2182) III; rmIs133[unc-54p::Q40::YFP] obtained by crossing IJ235 and IJ184.

CRISPR/Cas9 genome editing. mdt-15::degron::EmGFP knock-in was generated as described previously with modifications (Zhang et al., 2015). The degron::EmGFP repair template with homology arms (37 bp for 5' and 36 bp for 3') was amplified by PCR using pLZ29 as a template (Zhang et al., 2015), and the PCR products were purified by using PCR purification kit (QIAGEN, Germany). Wild-type adult worms were injected with pRF4 (roller injection marker, $50 \mathrm{ng} / \mu \mathrm{l})$, Cas9 protein $(250 \mathrm{ng} / \mu \mathrm{l}$, Integrated DNA Technologies, USA), tracrRNA (100 ng/ $\mu \mathrm{l}$, Integrated DNA Technologies, USA), $m d t-15$ crRNA (56 ng/ $\mu$ l, ATAATCTTAACTTGTAAGTT), and the purified degron::EmGFP repair template $(450 \mathrm{ng} / \mu \mathrm{l})$. Individual worms were subsequently transferred to new plates, and roller and non-roller F1 worms from plates that contained many rollers were genotyped by using PCR to identify worms that contained knock-in mutations. The $m d t-15::$ degron::EmGFP knock-in was confirmed by sequencing, and the strain 
was outcrossed four times to Lee lab N2 to remove potential background mutations. For generating $m d t-15(y h 8$, gof) (Svensk et al., 2013) in wild-type background, CRISPR/Cas9 plasmids targeting $m d t$ - 15 were generated by replacing pha-1 sgRNA sequence in pJW1285 (Ward 2015) with mdt-15 sgRNA sequence (TTTCTTGCCTGAGCTGATGT). Wild-type adults were injected with pRF4 (roller injection marker, $50 \mathrm{ng} / \mu \mathrm{l}$ ), pIJ285 (CRISPR/Cas9 plasmid targeting $m d t-15,50 \mathrm{ng} / \mu \mathrm{l})$, and repair template

\section{(ATCGAGCTCCTGTGCCTCCAGATCCACAACTAACATCAGCTCAGGCAAGAAATCCAC} CTGTTACCGTAGCA, $10 \mathrm{ng} / \mu \mathrm{l}$ ), and individual worms were transferred to new plates. F1 roller worms were genotyped by PCR to identify the $m d t$-15(gof) mutation. The mdt-15(gof) mutation was confirmed by using sequencing, and the $m d t-15(\mathrm{gof})$ mutant worms were outcrossed four times to wild-type worms to remove potential non-specific mutations.

Lifespan assays. Lifespan assays were performed as described previously with some modifications (Lee et al., 2010). Synchronized worms were cultured on the OP50-seeded NGM plates at different temperatures, and then transferred to plates containing $10 \mu \mathrm{M}$ 5-fluoro-2'deoxyuridine (FUdR, SIGMA, USA) at young adult stage (day 1) to prevent their progeny from hatching. Worms were transferred to fresh plates every other day until they stopped producing progeny for lifespan assays without FUdR treatment. Auxin-inducible degron assays were performed as described (Zhang et al., 2015). Briefly, synchronized worms were cultured on OP50-seeded NGM plates at $25^{\circ} \mathrm{C}$ or $15^{\circ} \mathrm{C}$ until reaching day 1 adult stage, and then transferred to new OP50-seeded NGM plates containing $1 \mathrm{mM}$ auxin (indole-3-acetic acid, ALFA AESAR, USA). Ethanol (solvent) was used as a control for auxin treatments. For temperature shift assays, worms were cultured at $20^{\circ} \mathrm{C}$ until reaching L4 stage, and subsequently shifted to $25^{\circ} \mathrm{C}$ or $15^{\circ} \mathrm{C}$. When the worms reached young adult stage (day 1), the worms were transferred to plates 
containing FUdR. For glucose-enriched diet experiments, 2\% glucose (Junsei Cheminal, Japan) was added to NGM as described previously (Lee et al., 2009). OP50 bacteria that were cultured overnight in liquid LB media were concentrated 20 times by centrifugation and seeded onto NGM plates containing $10 \mu \mathrm{g} / \mathrm{ml}$ kanamycin (SIGMA, USA) for dead bacteria assays as previously described (Lee et al., 2009). For RNAi experiments, bacteria that express double stranded RNA targeting a specific gene were cultured in liquid LB containing $50 \mu \mathrm{g} / \mathrm{ml}$ ampicillin (USB, USA) at $37^{\circ} \mathrm{C}$ overnight, and then seeded onto NGM containing $50 \mu \mathrm{g} / \mathrm{ml}$ ampicillin. The RNAi bacteria-seeded plates were incubated at $37^{\circ} \mathrm{C}$ overnight, and treated with $1 \mathrm{mM}$ isopropyl $\beta$-D-1-thiogalactopyranoside (IPTG, GOLDBIO, USA) to induce double stranded RNA at room temperature. Deaths of worms were determined by no response upon gently touching with a platinum wire. Animals that crawled off the plates, ruptured, bagged, or burrowed were censored but included in the statistical analysis. Days of adulthood are presented in lifespan curves, and time zero in lifespan data indicates a time when worms reached adulthood.

Body size assays. Wild-type and $m d t-15(t m 2182)$ worms were cultured on the OP50-seeded HG (high growth NGM) plates at $20^{\circ} \mathrm{C}$, and synchronized by using a bleaching method (Stiernagle, 2006). The bleached eggs were kept in M9 buffer at $20^{\circ} \mathrm{C}$ overnight, and then hatched L1 worms were cultured on OP50-seeded NGM plates at $25^{\circ} \mathrm{C}$ or at $15^{\circ} \mathrm{C}$. After becoming fully-grown adults $\left(72\right.$ hours at $25^{\circ} \mathrm{C}$ and 144 hours at $\left.15^{\circ} \mathrm{C}\right)$, the worms were placed on a $2 \%$ agarose pad and anesthetized by using $100 \mathrm{mM}$ sodium azide (SIGMA, USA). Bright field images were captured using AxioCam HRc (ZEISS, Germany) camera attached to a Zeiss Axioscope A.1 microscope (ZEISS, Germany). ImageJ (Schneider et al., 2012) was used for the quantification of body areas. 
Reproduction assays. Wild-type and $m d t-15(t m 2182)$ worms were cultured at $25^{\circ} \mathrm{C}$ or $15^{\circ} \mathrm{C}$ at least two generations. Single L2- or L3-staged larvae were transferred onto new OP50-seeded NGM plates and kept at $25^{\circ} \mathrm{C}$ or $15^{\circ} \mathrm{C}$. Twenty wild-type and thirty $m d t-15$ mutant worms at each temperature were used for one experimental set, and the assays were repeated five times. When the worms were producing eggs (for $2-3$ days at $25^{\circ} \mathrm{C}$ and $4-5$ days at $15^{\circ} \mathrm{C}$ ), the eggs were transferred onto new OP50-seeded NGM plates, and kept at $25^{\circ} \mathrm{C}$ or $15^{\circ} \mathrm{C}$. When the hatched larvae reached L3-L4 stages, larva/egg ratios were calculated for embryonic lethality. For $25^{\circ} \mathrm{C}$ and $16^{\circ} \mathrm{C}$ reproduction experiments, worms were first grown at $20^{\circ} \mathrm{C}$ to gravid adult stage. Ten worms were then transferred to $25^{\circ} \mathrm{C}$ and $16^{\circ} \mathrm{C}$, respectively, and allowed to lay eggs for 24 hours before being removed. The eggs remained at their respective temperatures for another 24 hours to allow them to hatch. The number of live progeny and dead eggs was then counted after another 24 hours.

RNA seq. analysis. HG-cultured wild-type and $m d t$-15(tm2182) worms were synchronized by using a bleaching method (Stiernagle, 2006) and incubated in M9 buffer at $20^{\circ} \mathrm{C}$ for overnight. The L1 worms were transferred onto OP50-seeded NGM plates and incubated at $25^{\circ} \mathrm{C}$ or $15^{\circ} \mathrm{C}$ until reaching day 1 adult stage. The adult worms were harvested by washing twice with M9 buffer, and then frozen at $-80^{\circ} \mathrm{C}$. Total RNA of the worms was extracted by using RNAiso plus (TAKARA, Japan). Three independent biological repeats were used for analysis. cDNA library was prepared and cDNA sequencing was performed by using a HiSeq 4000 platform by MACROGEN (MACROGEN, South Korea). Paired-end reads were aligned to the C. elegans genome ce11, and analyzed by using HISAT2 (v.2.0.5), StringTie (v.1.3.3), and Ballgown (v.2.0.0) methods (Pertea et al., 2016). R packages limma (v.3.24.15) (Ritchie et al., 2015) and edgeR (v.3.10.5) (Robinson et al., 2010) were used for analyzing differentially expressed genes 
(DEG), and genes whose expression was not detected (counts per million $(\mathrm{cpm})<1$ ) were excluded. Genes with significant changes in expression (fold change $>1.5$ and $p$ values $<0.05$ ) were further analyzed. Heat maps were generated by using Cluster 3.0 (de Hoon et al., 2004) and Java Treeview (Saldanha, 2004). Venn diagrams were generated by using Venn Diagram Plotter (http://omics.pnl.gov/software/venn-diagram-plotter). Gene ontology assays were performed by using DAVID (Huang da et al., 2009). Changes in the expression of metabolic genes were displayed as previously shown with modifications (Steinbaugh et al., 2015; Yu et al., 2017). Genes that were excluded during the DEG analysis are not presented in the metabolic pathways.

Quantitative RT-PCR. Quantitative RT-PCR was performed as described previously with modifications (Seo et al., 2013). Synchronized worms were cultured at different temperatures and were harvested at young adult stage (day 1) by washing twice with M9 buffer. paqr2(tm3410) and control (wild-type) worms were cultured at $20^{\circ} \mathrm{C}$ until reaching $\mathrm{L} 4$ stages, and then shifted to $25^{\circ} \mathrm{C}$ or $15^{\circ} \mathrm{C}$, for maintaining consistency with lifespan assays. Total RNA was isolated using RNAiso plus (TAKARA, Japan) and reverse transcription was performed using ImProm-II ${ }^{\mathrm{TM}}$ Reverse Transcriptase kit (PROMEGA, USA). Random primers (9-mers, Cosmogenetech, South Korea) were used for reverse transcription. Quantitative real time PCR was performed by using StepOne and StepOnePlus Real time PCR system (APPLIED BIOSYSTEMS, USA). Relative quantity of specific mRNA was analyzed by using comparative $\mathrm{Ct}$ methods described in the manufacturer's manual, and the mRNA level of ama-1, which encodes an RNA polymerase II large subunit, was used for normalization. The average of two technical repeats was used for each biological dataset.

\section{Primers used for quantitative RT-PCR assays.}


ama-1-F: TGGAACTCTGGAGTCACACC

ama-1-R: CATCCTCCTTCATTGAACGG

fat-2-F: AGTTTCTGGAGTTGCATGCGCTATC

fat-2-R: CAGCTTCGTAGACCTCAATATCCTC

fat-5-F: GTTCCAGAGGAAGAACTACCTCCCC

fat-5-R: GGGTGAAGCAGTAACGGAAGAGGGC

fat-6-F: GCGCTGCTCACTATTTCGGATGG

fat-6-R: GTGGGAATGTGTGATGGAAGTTGTG

fat-7-F: CTGCACGTCGCCGCAGCCATTG

fat-7-R: GAGAGCAAATGAGAAGACGGCC

hsp-16.1/11-F: CTCATGAGAGATATGGCTCAG

hsp-16.1/11-R: CATTGTTAACAATCTCAGAAG

F44E5.4/F44E5.5-F: GAATGGAAAGGTTGAGATCCTC

F44E5.4/F44E5.5-R: CCAACCAATCTTTCCGTATCTG

hsp-6-F: CTATGGGCCCAAAAGGAAGAAACGTG

hsp-6-R: GGGAATACACTTTTCCTTGAGCCTC

hsp-60-F: CTATGGGCCCAAAAGGAAGAAACGTG

hsp-60-R: GGATTTCGCGACGGTGACTCCGTCC

hsp-4-F: TCAGAAACTTCGCCGTGAGGT

hsp-4-R: AGAGTGACTCGATCTCGATCT

Oil red O staining. Oil Red O staining was performed as described previously with some modifications (O'Rourke et al., 2009). Synchronized worms were cultured on OP50-seeded NGM plates at $25^{\circ} \mathrm{C}$ or $15^{\circ} \mathrm{C}$. Approximately 300 young adult (day 1) worms were harvested by 
washing twice with M9 buffer and fixed with $60 \%$ isopropanol for two minutes. Oil Red O solution ( $0.5 \%$ in isopropanol, SIGMA, USA) was diluted in double distilled water $\left(\mathrm{ddH}_{2} \mathrm{O}\right)$ to prepare $60 \%$ working solution. Precipitates of Oil red $O$ were eliminated by filtering. The fixed worms were incubated in the $60 \%$ working solution overnight at $25^{\circ} \mathrm{C}$. The stained worms were washed with M9 buffer, and subsequently were placed on a 2\% agarose pad with M9 buffer containing $0.01 \%$ Triton X-100 (DAEJUNG, South Korea) using a micropipette. DIC images were captured using AxioCam HRc (ZEISS, Germany) camera attached to a Zeiss Axioscope A.1 microscope (ZEISS, Germany). ImageJ (Schneider et al., 2012) was used for the quantification of Oil Red O intensity. The backgrounds of the images were subtracted and the images were then converted to 8-bit grayscale images. Same thresholds were set in the same experimental sets for detecting the Oil red O signals. Areas that had higher intensities than the threshold in the whole body were measured, and the areas were normalized by body size.

Fluorescence imaging. GFP transgenic worms were anesthetized by using $100 \mathrm{mM}$ sodium azide, and then placed on a $2 \%$ agarose pad. Images of the worms were captured by using AxioCam HRc (ZEISS, Germany) camera attached to a Zeiss Axioscope A.1 microscope (ZEISS, Germany). ImageJ (Schneider et al., 2012) was used to quantify the fluorescence intensity and the background signals were subtracted. High resolution confocal images were captured by using the Nikon A1si/Ni-E upright confocal microscope with 60×, 1.4 NA oil-immersion objective lens in the Advanced Neural Imaging Center in Korea Brain Research Institute (KBRI). For double RNAi treatments, control and gene-specific RNAi bacteria were separately cultured in liquid LB containing $50 \mu \mathrm{g} / \mathrm{ml}$ ampicillin (USB, USA) at $37^{\circ} \mathrm{C}$. OD590 was adjusted to 0.9 , and same volumes of the bacterial culture were mixed as described previously (Rea et al., 2007). Genespecific RNAi bacteria were mixed with control RNAi bacteria for single RNAi treatments in the 
same experimental sets for comparison.

Fatty acid composition assays. Lipid extraction was performed as described previously with some modifications (Deline et al., 2013). Wild-type and $m d t-15(t m 2182)$ worms were synchronized by using a bleaching method (Stiernagle, 2006) and incubated in M9 solution for overnight at $20^{\circ} \mathrm{C}$. Hatched L1 worms were then cultured on OP50-seeded NGM plates at $25^{\circ} \mathrm{C}$ or $15^{\circ} \mathrm{C}$. Approximately $1000-1500$ day 1 adult worms were harvested and washed three times with $\mathrm{ddH}_{2} \mathrm{O}$. The harvested worms were frozen in liquid nitrogen and stored at $-80^{\circ} \mathrm{C}$ until use. Fatty acid methyl esters (FAMEs) were prepared by adding $2 \mathrm{ml}$ of sulfuric acid $(2.5 \%) /$ methanol solution to the worm samples, followed by heating at $70^{\circ} \mathrm{C}$ for one hour. The

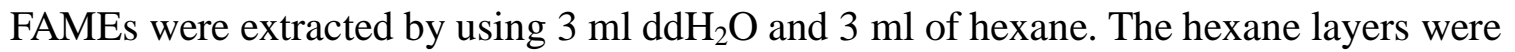
evaporated and analyzed by using GC/MS (GCMS-QP2010, Shimadzu; HP-INOWAX capillary column, $30 \mathrm{~m}, 0.25 \mathrm{~mm}$, Agilent).

Statistics. Statistics used for each experiment was described in Figure Legends. Briefly, $p$ values were calculated by using two-tailed Student t-test for embryonic lethality, body size, RNA-seq., qRT-PCR, fluorescence imaging, Oil red O, and GC/MS assays. For survival assays, statistical analysis was performed by using OASIS 2 (online application of survival analysis, http://sbi.postech.ac.kr/oasis2) (Han et al., 2016) and $p$ values were calculated by using log-rank (Mantel-Cox method) test. Significance of the overlaps among gene sets was calculated by using hypergeometric probability test (http://systems.crump.ucla.edu/hypergeometric/index.php, provided by Graeber lab).

\section{Acknowledgments}


We thank all Lee laboratory members for help and discussion. We also thank Craig Mello lab for providing CRISPR/Cas9 protocol, and Marc Pilon and Junho Lee labs for strains. This work was supported by the Korean Government (MSIP) through the National Research Foundation of Korea (NRF) (NRF-2016R1E1A1A01941152) to S-J. V. L. and by Canadian Institutes of Health Research (CIHR) (PJT-153199) to S.T.

\section{Author contributions}

D.L. contributed to designing and performing the majority of experiments for all the assays described in the manuscript, data analysis, and writing manuscript; S.W.A.A. and Y.J. contributed to survival assay experiments; Y.Y. and Y.L. contributed to lipid composition analysis; Y.R. and C.M.H. contributed to confocal microscopy imaging; D.M. contributed to RNA-seq. analysis; G.Y.S.G, A.B., and S.T. contributed to reproduction assay experiments; S.-J.V.L. contributed to designing all experiments, data analysis, and writing manuscript.

\section{References}

Allen, B.L., and Taatjes, D.J. 2015. The Mediator complex: a central integrator of transcription. Nature reviews Molecular cell biology. doi: 10.1038/nrm3951

Artan, M., Jeong, D.E., Lee, D., Kim, Y.I., Son, H.G., Husain, Z., Kim, J., Altintas, O., Kim, K., Alcedo, J., et al. 2016. Food-derived sensory cues modulate longevity via distinct neuroendocrine insulin-like peptides. Genes \& development. doi: 10.1101/gad.279448.116 Brock, T.J., Browse, J., and Watts, J.L. 2007. Fatty acid desaturation and the regulation of 
adiposity in Caenorhabditis elegans. Genetics. doi: 10.1534/genetics.107.071860

Brokate-Llanos, A.M., Garzon, A., and Munoz, M.J. 2014. Escherichia coli carbon source metabolism affects longevity of its predator Caenorhabditis elegans. Mechanisms of ageing and development. doi: 10.1016/j.mad.2014.09.001

Brunquell, J., Morris, S., Lu, Y., Cheng, F., and Westerheide, S.D. 2016. The genome-wide role of HSF-1 in the regulation of gene expression in Caenorhabditis elegans. BMC genomics. doi: $10.1186 / \mathrm{s} 12864-016-2837-5$

Chen, Y.C., Chen, H.J., Tseng, W.C., Hsu, J.M., Huang, T.T., Chen, C.H., and Pan, C.L. 2016. A C. elegans Thermosensory Circuit Regulates Longevity through crh-1/CREB-Dependent flp-6 Neuropeptide Signaling. Developmental cell. doi: 10.1016/j.devcel.2016.08.021

Chi, Y., and Gupta, R.K. 1998. Alterations in membrane fatty acid unsaturation and chain length in hypertension as observed by 1H NMR spectroscopy. American journal of hypertension. doi: Conti, B. 2008. Considerations on temperature, longevity and aging. Cellular and molecular life sciences : CMLS. doi: 10.1007/s00018-008-7536-1

Conti, B., Sanchez-Alavez, M., Winsky-Sommerer, R., Morale, M.C., Lucero, J., Brownell, S., Fabre, V., Huitron-Resendiz, S., Henriksen, S., Zorrilla, E.P., et al. 2006. Transgenic mice with a reduced core body temperature have an increased life span. Science (New York, NY). doi:

\subsection{6/science.1132191}

de Hoon, M.J., Imoto, S., Nolan, J., and Miyano, S. 2004. Open source clustering software. Bioinformatics (Oxford, England). doi: 10.1093/bioinformatics/bth078

Deline, M.L., Vrablik, T.L., and Watts, J.L. 2013. Dietary supplementation of polyunsaturated fatty acids in Caenorhabditis elegans. Journal of visualized experiments : JoVE. doi:

$10.3791 / 50879$ 
Devkota, R., Svensk, E., Ruiz, M., Stahlman, M., Boren, J., and Pilon, M. 2017. The adiponectin receptor AdipoR2 and its Caenorhabditis elegans homolog PAQR-2 prevent membrane rigidification by exogenous saturated fatty acids. PLoS genetics. doi:

10.1371/journal.pgen.1007004

Goh, G.Y., Martelli, K.L., Parhar, K.S., Kwong, A.W., Wong, M.A., Mah, A., Hou, N.S., and Taubert, S. 2014. The conserved Mediator subunit MDT-15 is required for oxidative stress responses in Caenorhabditis elegans. Aging cell. doi: 10.1111/acel.12154

Grants, J.M., Goh, G.Y., and Taubert, S. 2015. The Mediator complex of Caenorhabditis elegans: insights into the developmental and physiological roles of a conserved transcriptional coregulator. Nucleic acids research. doi: 10.1093/nar/gkv037

Han, S., Schroeder, E.A., Silva-Garcia, C.G., Hebestreit, K., Mair, W.B., and Brunet, A. 2017. Mono-unsaturated fatty acids link H3K4me3 modifiers to C. elegans lifespan. Nature. doi: $10.1038 /$ nature21686

Han, S.K., Lee, D., Lee, H., Kim, D., Son, H.G., Yang, J.S., Lee, S.V., and Kim, S. 2016. OASIS 2: online application for survival analysis 2 with features for the analysis of maximal lifespan and healthspan in aging research. Oncotarget. doi: 10.18632/oncotarget.11269

Heintz, C., Doktor, T.K., Lanjuin, A., Escoubas, C., Zhang, Y., Weir, H.J., Dutta, S., Silva-Garcia, C.G., Bruun, G.H., Morantte, I., et al. 2017. Splicing factor 1 modulates dietary restriction and TORC1 pathway longevity in C. elegans. Nature. doi: 10.1038/nature20789

Higuchi-Sanabria, R., Frankino, P.A., Paul, J.W., 3rd, Tronnes, S.U., and Dillin, A. 2018. A Futile Battle? Protein Quality Control and the Stress of Aging. Developmental cell. doi:

10.1016/j.devcel.2017.12.020

Holthuis, J.C., and Menon, A.K. 2014. Lipid landscapes and pipelines in membrane homeostasis. 
Nature. doi: 10.1038/nature13474

Horikawa, M., Sural, S., Hsu, A.L., and Antebi, A. 2015. Co-chaperone p23 regulates C. elegans Lifespan in Response to Temperature. PLoS genetics. doi: 10.1371/journal.pgen.1005023

Huang da, W., Sherman, B.T., and Lempicki, R.A. 2009. Systematic and integrative analysis of large gene lists using DAVID bioinformatics resources. Nature protocols. doi: 10.1038/nprot.2008.211

Jeong, D.E., Artan, M., Seo, K., and Lee, S.J. 2012. Regulation of lifespan by chemosensory and thermosensory systems: findings in invertebrates and their implications in mammalian aging. Frontiers in genetics. doi: 10.3389/fgene.2012.00218

Kim, H.E., Grant, A.R., Simic, M.S., Kohnz, R.A., Nomura, D.K., Durieux, J., Riera, C.E., Sanchez, M., Kapernick, E., Wolff, S., et al. 2016. Lipid Biosynthesis Coordinates a Mitochondrial-to-Cytosolic Stress Response. Cell. doi: 10.1016/j.cell.2016.08.027

Labbadia, J., Brielmann, R.M., Neto, M.F., Lin, Y.F., Haynes, C.M., and Morimoto, R.I. 2017. Mitochondrial Stress Restores the Heat Shock Response and Prevents Proteostasis Collapse during Aging. Cell reports. doi: 10.1016/j.celrep.2017.10.038

Lee, D., Jeong, D.E., Son, H.G., Yamaoka, Y., Kim, H., Seo, K., Khan, A.A., Roh, T.Y., Moon, D.W., Lee, Y., et al. 2015. SREBP and MDT-15 protect $C$. elegans from glucose-induced accelerated aging by preventing accumulation of saturated fat. Genes \& development. doi: 10.1101/gad.266304.115

Lee, S.J., Hwang, A.B., and Kenyon, C. 2010. Inhibition of respiration extends C. elegans life span via reactive oxygen species that increase HIF-1 activity. Current biology : CB. doi: 10.1016/j.cub.2010.10.057

Lee, S.J., and Kenyon, C. 2009. Regulation of the longevity response to temperature by 
thermosensory neurons in Caenorhabditis elegans. Current biology : CB. doi:

10.1016/j.cub.2009.03.041

Lee, S.J., Murphy, C.T., and Kenyon, C. 2009. Glucose shortens the life span of C. elegans by downregulating DAF-16/FOXO activity and aquaporin gene expression. Cell metabolism. doi: 10.1016/j.cmet.2009.10.003

Lopez-Otin, C., Blasco, M.A., Partridge, L., Serrano, M., and Kroemer, G. 2013. The hallmarks of aging. Cell. doi: 10.1016/j.cell.2013.05.039

Ma, D.K., Li, Z., Lu, A.Y., Sun, F., Chen, S., Rothe, M., Menzel, R., Sun, F., and Horvitz, H.R. 2015. Acyl-CoA Dehydrogenase Drives Heat Adaptation by Sequestering Fatty Acids. Cell. doi: 10.1016/j.cell.2015.04.026

McKay, R.M., McKay, J.P., Avery, L., and Graff, J.M. 2003. C elegans: a model for exploring the genetics of fat storage. Developmental cell. doi: 10.1016/S1534-5807(02)00411-2

Morley, J.F., Brignull, H.R., Weyers, J.J., and Morimoto, R.I. 2002. The threshold for polyglutamine-expansion protein aggregation and cellular toxicity is dynamic and influenced by aging in Caenorhabditis elegans. Proceedings of the National Academy of Sciences of the United States of America. doi: 10.1073/pnas.152161099

Murphy, C.T., McCarroll, S.A., Bargmann, C.I., Fraser, A., Kamath, R.S., Ahringer, J., Li, H., and Kenyon, C. 2003. Genes that act downstream of DAF-16 to influence the lifespan of Caenorhabditis elegans. Nature. doi: 10.1038/nature01789

Murray, P., Hayward, S.A., Govan, G.G., Gracey, A.Y., and Cossins, A.R. 2007. An explicit test of the phospholipid saturation hypothesis of acquired cold tolerance in Caenorhabditis elegans. Proceedings of the National Academy of Sciences of the United States of America. doi: 10.1073/pnas.0609590104 
O'Rourke, E.J., Kuballa, P., Xavier, R., and Ruvkun, G. 2013. omega-6 Polyunsaturated fatty acids extend life span through the activation of autophagy. Genes \& development. doi:

10.1101/gad.205294.112

O'Rourke, E.J., Soukas, A.A., Carr, C.E., and Ruvkun, G. 2009. C. elegans major fats are stored in vesicles distinct from lysosome-related organelles. Cell metabolism. doi:

10.1016/j.cmet.2009.10.002

Pang, S., Lynn, D.A., Lo, J.Y., Paek, J., and Curran, S.P. 2014. SKN-1 and Nrf2 couples proline catabolism with lipid metabolism during nutrient deprivation. Nature communications. doi:

$10.1038 /$ ncomms6048

Pertea, M., Kim, D., Pertea, G.M., Leek, J.T., and Salzberg, S.L. 2016. Transcript-level expression analysis of RNA-seq experiments with HISAT, StringTie and Ballgown. Nature protocols. doi: 10.1038/nprot.2016.095

Pukkila-Worley, R., Feinbaum, R.L., McEwan, D.L., Conery, A.L., and Ausubel, F.M. 2014. The evolutionarily conserved mediator subunit MDT-15/MED15 links protective innate immune responses and xenobiotic detoxification. PLoS pathogens. doi: 10.1371/journal.ppat.1004143 Rea, S.L., Ventura, N., and Johnson, T.E. 2007. Relationship between mitochondrial electron transport chain dysfunction, development, and life extension in Caenorhabditis elegans. PLoS biology. doi: 10.1371/journal.pbio.0050259

Ritchie, M.E., Phipson, B., Wu, D., Hu, Y., Law, C.W., Shi, W., and Smyth, G.K. 2015. limma powers differential expression analyses for RNA-sequencing and microarray studies. Nucleic acids research. doi: 10.1093/nar/gkv007

Robinson, M.D., McCarthy, D.J., and Smyth, G.K. 2010. edgeR: a Bioconductor package for differential expression analysis of digital gene expression data. Bioinformatics (Oxford, 
England). doi: 10.1093/bioinformatics/btp616

Saldanha, A.J. 2004. Java Treeview--extensible visualization of microarray data. Bioinformatics (Oxford, England). doi: 10.1093/bioinformatics/bth349

Schleit, J., Wall, V.Z., Simko, M., and Kaeberlein, M. 2011. The MDT-15 subunit of mediator interacts with dietary restriction to modulate longevity and fluoranthene toxicity in Caenorhabditis elegans. PloS one. doi: 10.1371/journal.pone.0028036

Schneider, C.A., Rasband, W.S., and Eliceiri, K.W. 2012. NIH Image to ImageJ: 25 years of image analysis. Nature methods. doi: 10.1038/nmeth.2089

Seo, K., Choi, E., Lee, D., Jeong, D.E., Jang, S.K., and Lee, S.J. 2013. Heat shock factor 1 mediates the longevity conferred by inhibition of TOR and insulin/IGF-1 signaling pathways in C. elegans. Aging cell. doi: 10.1111/acel.12140

Soderberg, M., Edlund, C., Kristensson, K., and Dallner, G. 1991. Fatty acid composition of brain phospholipids in aging and in Alzheimer's disease. Lipids. doi:

Son, H.G., and Lee, S.V. 2017. Longevity regulation by NMD-mediated mRNA quality control. BMB reports. doi: 10.5483/BMBRep.2017.50.4.045

Son, H.G., Seo, M., Ham, S., Hwang, W., Lee, D., An, S.W., Artan, M., Seo, K., Kaletsky, R., Arey, R.N., et al. 2017. RNA surveillance via nonsense-mediated mRNA decay is crucial for longevity in $d a f-2 /$ insulin/IGF-1 mutant $C$. elegans. Nature communications. doi:

\subsection{8/ncomms14749}

Steinbaugh, M.J., Narasimhan, S.D., Robida-Stubbs, S., Moronetti Mazzeo, L.E., Dreyfuss, J.M., Hourihan, J.M., Raghavan, P., Operana, T.N., Esmaillie, R., and Blackwell, T.K. 2015. Lipidmediated regulation of $\mathrm{SKN}-1 / \mathrm{Nrf}$ in response to germ cell absence. eLife. doi:

10.7554/eLife.07836 
Stiernagle, T. 2006. Maintenance of C. elegans. WormBook : the online review of C elegans biology. doi: 10.1895/wormbook.1.101.1

Svensk, E., Devkota, R., Stahlman, M., Ranji, P., Rauthan, M., Magnusson, F., Hammarsten, S., Johansson, M., Boren, J., and Pilon, M. 2016. Caenorhabditis elegans PAQR-2 and IGLR-2 Protect against Glucose Toxicity by Modulating Membrane Lipid Composition. PLoS genetics. doi: 10.1371/journal.pgen.1005982

Svensk, E., Stahlman, M., Andersson, C.H., Johansson, M., Boren, J., and Pilon, M. 2013.

PAQR-2 regulates fatty acid desaturation during cold adaptation in C. elegans. PLoS genetics. doi: 10.1371/journal.pgen.1003801

Sztolsztener, M.E., Dobrzyn, A., Pikula, S., Tylki-Szymanska, A., and Bandorowicz-Pikula, J. 2012. Impaired dynamics of the late endosome/lysosome compartment in human Niemann-Pick type C skin fibroblasts carrying mutation in NPC1 gene. Molecular bioSystems. doi:

\section{$10.1039 / \mathrm{c} 2 \mathrm{mb} 05447 \mathrm{~g}$}

Tabrez, S.S., Sharma, R.D., Jain, V., Siddiqui, A.A., and Mukhopadhyay, A. 2017. Differential alternative splicing coupled to nonsense-mediated decay of mRNA ensures dietary restrictioninduced longevity. Nature communications. doi: 10.1038/s41467-017-00370-5

Tanaka, T., Ikita, K., Ashida, T., Motoyama, Y., Yamaguchi, Y., and Satouchi, K. 1996. Effects of growth temperature on the fatty acid composition of the free-living nematode Caenorhabditis elegans. Lipids. doi: 10.1007/BF02524292

Taubert, S., Hansen, M., Van Gilst, M.R., Cooper, S.B., and Yamamoto, K.R. 2008. The Mediator subunit MDT-15 confers metabolic adaptation to ingested material. PLoS genetics. doi: 10.1371/journal.pgen.1000021

Taubert, S., Van Gilst, M.R., Hansen, M., and Yamamoto, K.R. 2006. A Mediator subunit, MDT- 
15, integrates regulation of fatty acid metabolism by NHR-49-dependent and -independent pathways in C. elegans. Genes \& development. doi: 10.1101/gad.1395406

Van Gilst, M.R., Hadjivassiliou, H., Jolly, A., and Yamamoto, K.R. 2005. Nuclear hormone receptor NHR-49 controls fat consumption and fatty acid composition in C. elegans. PLoS biology. doi: 10.1371/journal.pbio.0030053

Wang, L., Folsom, A.R., and Eckfeldt, J.H. 2003. Plasma fatty acid composition and incidence of coronary heart disease in middle aged adults: the Atherosclerosis Risk in Communities (ARIC) Study. Nutrition, metabolism, and cardiovascular diseases : NMCD. doi: 10.1016/S09394753(03)80029-7

Xiao, R., Zhang, B., Dong, Y., Gong, J., Xu, T., Liu, J., and Xu, X.Z. 2013. A genetic program promotes $C$. elegans longevity at cold temperatures via a thermosensitive TRP channel. Cell. doi: 10.1016/j.cell.2013.01.020

Yang, F., Vought, B.W., Satterlee, J.S., Walker, A.K., Jim Sun, Z.Y., Watts, J.L., DeBeaumont, R., Saito, R.M., Hyberts, S.G., Yang, S., et al. 2006. An ARC/Mediator subunit required for SREBP control of cholesterol and lipid homeostasis. Nature. doi: 10.1038/nature04942 Yu, Y., Mutlu, A.S., Liu, H., and Wang, M.C. 2017. High-throughput screens using photohighlighting discover BMP signaling in mitochondrial lipid oxidation. Nature communications. doi: 10.1038/s41467-017-00944-3

Zhang, L., Ward, J.D., Cheng, Z., and Dernburg, A.F. 2015. The auxin-inducible degradation (AID) system enables versatile conditional protein depletion in C. elegans. Development (Cambridge, England). doi: 10.1242/dev.129635 
bioRxiv preprint doi: https://doi.org/10.1101/366153; this version posted July 10,2018 . The copyright holder for this preprint (which was not certified by peer review) is the author/funder. All rights reserved. No reuse allowed without permission. 
Figure 1. MDT-15 limits the body size and longevity at low temperatures. (A) Lifespan of wild-type and $m d t-15(t m 2182)[m d t-15(-)] C$. elegans at $25^{\circ} \mathrm{C}$ and $15^{\circ} \mathrm{C}$. Worms were cultured at indicated temperature from hatching. The lifespan assays in panel $\mathbf{A}$ were performed without 5-fluoro-2'-deoxyuridine (FUdR), which prevents progeny from hatching. (B) Expression of $m d t$ 15::degron::EmGFP generated by CRISPR/Cas9 knock-in in the nuclei of many tissues, including neurons, intestine and hypodermis at $20^{\circ} \mathrm{C}$. (C) Depletion of $M D T$ 15::degron::EmGFP by treatment with auxin at $15^{\circ} \mathrm{C}$ was captured by using confocal microscopy. The worms carry ubiquitously expressed TIR1 (eft-3p::TIR1::mRuby) for the auxininducible protein degradation. (D) MDT-15 depletion substantially decreased longevity at $15^{\circ} \mathrm{C}$. Control and MDT-15 depletion indicate solvent (ethanol) and auxin treatments, respectively. (E) Lifespan curves of wild-type and $m d t-15(y h 8)[m d t-15($ gof $)]$ mutant animals at $25^{\circ} \mathrm{C}$ and $15^{\circ} \mathrm{C}$. (F) The relative ratio of body areas of $m d t-15(-)$ and wild-type animals at $25^{\circ} \mathrm{C}$ and $15^{\circ} \mathrm{C}$. The relative ratios were calculated from the same data set shown in Supplementary Fig. S1E. Bars indicate the averages of the ratios from three independent experiments. Eight worms were analyzed in each experimental set. Error bars represent standard error of the mean (SEM) (twotailed Student's t-tests, $\left.{ }^{*} p<0.05\right)$. (G) Images represent unhatched progeny in $m d t-15(-)$ at $15^{\circ} \mathrm{C}$. (H) Percent wild-type and $m d t-15(-)$ mutant larvae that hatched at $25^{\circ} \mathrm{C}$ and $15^{\circ} \mathrm{C}$. The hatching defect caused by $m d t-15(-)$ was also measured at $16^{\circ} \mathrm{C}$ (Supplementary Fig. S1F). Error bars represent SEM (two-tailed Student's t-tests, ${ }^{* * *} p<0.001, \mathrm{n}=248$ for wild-type at $25^{\circ} \mathrm{C}, \mathrm{n}=250$ for wild-type at $15^{\circ} \mathrm{C}, \mathrm{n}=644$ for $m d t-15(-)$ at $25^{\circ} \mathrm{C}$, and $\mathrm{n}=1524$ for $m d t-15(-)$ at $15^{\circ} \mathrm{C}$ from five independent experiments). We were able to maintain $m d t-15(-)$ worms at low temperatures, as the embryonic lethality was not $100 \%$. See Supplementary Table 1 for statistical analysis and additional repeats of the lifespan assays. 
Figure 2. Genes that are differentially regulated by MDT-15 at different temperatures. (A-

C) Volcano plots represent genes up- and down-regulated in wild-type at $15^{\circ} \mathrm{C}(\mathbf{A})$, and genes

up-regulated at $15^{\circ} \mathrm{C}(\mathbf{B})$ and down-regulated at $15^{\circ} \mathrm{C}(\mathbf{C})$ in an MDT-15 dependent manner. (D,

E) Gene ontology (GO) terms enriched among the genes up-regulated at $15^{\circ} \mathrm{C}(\mathbf{D})$ and down-

regulated at $15^{\circ} \mathrm{C}(\mathbf{E})$ in an MDT-15-dependent manner. 
Figure 3. The effects of temperature changes and $m d t-15(-)$ mutations on the expression of genes involved in metabolism. (A) Simplified carbohydrate and lipid metabolic pathways. Changes in the expression of genes involved in carbohydrate and fatty acid metabolism are shown. Fold changes at $15^{\circ} \mathrm{C}$ and by $m d t-15(\mathrm{tm} 2182)[m d t-15(-)]$ mutations are shown in circles and squares, respectively. GPI: glucose-6-phosphate isomerase; PFK: phospho-fructo-kinase; TPI: triosephosphate isomerase; GAPDH: glyceraldehyde 3-phosphate dehydrogenase; PGK: phosphoglycerate kinase; PGM: phosphoglycerate mutase; PK: pyruvate kinase; CS: citrate synthase; IDH: isocitrate dehydrogenase; $\alpha$-KGDH: $\alpha$-ketoglutarate dehydrogenase; SCS: succinyl-CoA synthetase; SDH: succinate dehydrogenase; MDH: malate dehydrogenase; ACC: acetyl-CoA carboxylase; FAS: fatty acid synthase; FAT: fatty acid desaturase; ELO: fatty acid elongase; LBP: lipid-binding protein; FAR: fatty acid- and retinol-binding protein; ACS: acylCoA synthetase; CPT: carnitine palmitoyl transferase; ACO: acyl-CoA oxidase; ACDH: acylCoA dehydrogenase; ECH: enoyl-CoA hydratase; HACD: hydroxyacyl-CoA dehydrogenase. (B) mRNA levels of seven fatty acid desaturases in wild-type and $m d t-15(-)$ at $25^{\circ} \mathrm{C}$ and $15^{\circ} \mathrm{C}$ were analyzed from RNA seq. data. (C) mRNA levels of fat-7 in wild-type and $m d t-15(-)$ at $25^{\circ} \mathrm{C}$ and $15^{\circ} \mathrm{C}$ were measured by using quantitative RT-PCR (qRT-PCR) (N=9, biological replicates). (D) Quantified fluorescence intensity of fat-7::GFP in wild-type and $m d t-15(-)$ animals at $25^{\circ} \mathrm{C}$ and $15^{\circ} \mathrm{C}$. Representative images are shown in Supplementary Fig. taG. Error bars represent SEM (two-tailed Student's $t$-tests, ${ }^{*} p<0.05,{ }^{*} p<0.01,{ }^{*} * *<0.001, \mathrm{n}=21$ for wild-type at $25^{\circ} \mathrm{C}$, wildtype at $15^{\circ} \mathrm{C}, m d t-15(-)$ at $25^{\circ} \mathrm{C}$, and $\mathrm{n}=20$ for $m d t-15(-)$ at $15^{\circ} \mathrm{C}$ from three independent experiments) 
Figure 4. MDT-15 increases overall fat levels and UFA/SFA ratios at low temperatures. (A) Oil red O signals represent overall fat levels in wild-type and $m d t-15(t m 2182)$ [mdt-15(-)] mutant animals at $25^{\circ} \mathrm{C}$ and $15^{\circ} \mathrm{C}$. (B) Quantification of the Oil red O staining in panel A. Error bars represent SEM (two-tailed Student's $t$-tests, $* * * p<0.001, \mathrm{n}=20$ for wild-type at $25^{\circ} \mathrm{C}, \mathrm{n}=23$ for wild-type at $15^{\circ} \mathrm{C}, \mathrm{n}=20$ for $m d t-15(-)$ at $25^{\circ} \mathrm{C}$, and $\mathrm{n}=24$ for $m d t-15(-)$ at $15^{\circ} \mathrm{C}$ from three independent experiments). (C) Bright field images of wild-type and $m d t-15(-)$ mutants at $25^{\circ} \mathrm{C}$ and $15^{\circ} \mathrm{C} \cdot m d t-15(-)$ mutant animals displayed pale intestines at $15^{\circ} \mathrm{C}$. The images were taken when the worms reached day 1 adult stages. (D) Total fatty acid composition in wild-type animals at $25^{\circ} \mathrm{C}$ and $15^{\circ} \mathrm{C}$ was analyzed by using gas chromatography/mass spectrometry (GC/MS) (N=5, biological replicates). Fractions of each fatty acid in the total fatty acids (mole/mole) were calculated. (E, F) Fractions of saturated fatty acids (E) and unsaturated fatty acids $(\mathbf{F})$ in wild-type and $m d t-15(-)$ mutant animals at $25^{\circ} \mathrm{C}$ and $15^{\circ} \mathrm{C}$. (G) The ratios of unsaturated fatty acids/saturated fatty acids that were calculated from the panels $\mathbf{E}$ and $\mathbf{F}$. Error bars represent SEM (two-tailed Student's $t$-tests, ${ }^{*} p<0.05, * * p<0.01, * * * p<0.001, \mathrm{n}=5$ ). 
Figure 5. Maintenance of UFA/SFA ratios permits long lifespan at low temperatures. (A) Lifespan curves of wild-type and fat-6(tm331); fat-7(wa36) [fat-6(-); fat-7(-)] mutants at $15^{\circ} \mathrm{C}$ and $25^{\circ} \mathrm{C}$. Wild-type and fat-6(-); fat-7(-) mutants were cultured at $20^{\circ} \mathrm{C}$ until reaching $\mathrm{L} 4$ larval stages and then shifted to $15^{\circ} \mathrm{C}$ or $25^{\circ} \mathrm{C} \cdot m d t-15(\mathrm{tm} 2182)$ [mdt-15(-)] mutations also substantially suppressed longevity at $15^{\circ} \mathrm{C}$, when the worms were shifted from $20^{\circ} \mathrm{C}$ to $15^{\circ} \mathrm{C}$ at L4 larval stages (Supplementary Fig. S1A). (B) Lifespan curves of wild-type and paqr-2(tm3410) [paqr-2(-)] animals at $25^{\circ} \mathrm{C}$ and $15^{\circ} \mathrm{C}$. Wild-type and paqr-2(-) worms were shifted from $20^{\circ} \mathrm{C}$ to indicated temperatures at L4 stages. (C) Lifespan curves of wild-type and $n h r-49(g k 405)[n h r$ $49(-)]$ animals at $25^{\circ} \mathrm{C}$ and $15^{\circ} \mathrm{C}$. (D) $n h r-49(-)$ mutations did not further shorten the lifespan of $m d t-15(-)$ worms at $15^{\circ} \mathrm{C}$. Genetic inhibition of $s k n-1$ or $s b p-1$, which encodes a transcription factor that interacts with MDT-15 (Goh et al., 2014; Pang et al., 2014; Yang et al., 2006), had a small or no effect on the longevity at low temperatures (Supplementary Fig. S3D, E). (E) Glucose-enriched diets (2\%) substantially suppressed the longevity of wild-type animals at $15^{\circ} \mathrm{C}$. (F) Lifespan curves of worms fed with dead bacteria supplemented with $2 \%$ glucose at $25^{\circ} \mathrm{C}$ and $15^{\circ} \mathrm{C}$. These data indicate that the shortened lifespan upon glucose was not caused by changes in bacterial metabolism on glucose-rich media at different temperatures. The worms were shifted from $20^{\circ} \mathrm{C}$ to $25^{\circ} \mathrm{C}$ or $15^{\circ} \mathrm{C}$ at $\mathrm{L} 4$ stages. See Supplementary Table 1 for statistical analysis and additional repeats of the lifespan assays. 
Figure 6. MDT-15 is required for maintaining proteostasis at low temperatures. (A) mRNA levels of selected cytosolic chaperones, hsp-16.1, hsp-16.11, hsp-16.49, hsp-16.48, hsp-16.41, $h s p-16.2, F 44 E 5.4$, and F44E5.5 in wild-type and $m d t-15(-)$ worms at $25^{\circ} \mathrm{C}$ and $15^{\circ} \mathrm{C}$ were measured by using RNA seq ( $\mathrm{N}=3$, biological replicates). Note that the mRNA levels of $h s p-16.1$ and $h s p-16.11$, those of $h s p-16.48$ and $h s p-16.49$, and those of F44E5.4 and F44R5.5 may not be completely distinguishable, because the sequences of these pairs of transcripts are very similar. The induction of some of these chaperone genes by inhibition of $m d t-15$ was also observed at $20^{\circ} \mathrm{C}$ in a previous report (Taubert et al., 2008). (B) Images of $h s p-16.1:: G F P$ transgenic worms treated with control RNAi or $m d t-15$ RNAi at $15^{\circ} \mathrm{C}$. (C) mRNA levels of $h s p-16.1 / 11$ were measured by using qRT-PCR in wild-type and $m d t-15(\mathrm{tm} 2182)[m d t-15(-)]$ animals at $15^{\circ} \mathrm{C}$ ( $\mathrm{N}=7$, biological replicates). (D) The expression of $F 44 E 5.4 / 5$ at $15^{\circ} \mathrm{C}$ was determined by using qRT-PCR (N=6, biological replicates). (E-G) qRT-PCR data represent mRNA levels of $h s p-6$ ( $\mathrm{N}=6$, biological replicates) $(\mathbf{E}), h s p-60(\mathrm{~N}=6$, biological replicates) $(\mathbf{F})$, and $h s p-4(\mathrm{~N}=5$, biological replicates) $(\mathbf{G})$ in wild-type and $m d t-15(-)$ worms at $15^{\circ} \mathrm{C}$. (H) Fluorescence images of Q40::YFP transgenic worms in wild-type and $m d t-15(-)$ backgrounds at $15^{\circ} \mathrm{C}$. The images were captures at L4 larval stages. (I) Quantification of the Q40::YFP aggregates shown in panel $\mathbf{H}$ ( $\mathrm{n}=30$ for all conditions from three independent experiments). (J) Fluorescence images of $h s p$ 16.1::GFP treated with $m d t-15 \mathrm{RNAi}$ or $m d t-15 / h s f-1$ double RNAi at $15^{\circ} \mathrm{C}$. (K) Images of Q40::YFP transgenic worms treated with $m d t$-15 RNAi, $h s f-1$ RNAi, or $m d t-15 / h s f-1$ double RNAi at $15^{\circ} \mathrm{C}$. The images were taken at L4 stages. (L) Quantification of the Q40::YFP aggregates shown in panel $\mathbf{K}$ ( $\mathrm{n}=29$ for control RNAi, and $\mathrm{n}=28$ for $m d t$ - 15 RNAi, $h s f-1$ RNAi, and $m d t-15 / h s f-1$ RNAi from three independent experiments). Note that the number of Q40::YFP aggregation in control RNAi condition was smaller than wild-type condition in panel $\mathbf{I}$. We 
speculate that different food types (OP50 vs. HT115) affect Q40 aggregation. Error bars

represent SEM (two-tailed Student's $t$-tests, ${ }^{*} p<0.05, *^{*} p<0.01$, $*^{* *} p<0.001$, n.s.: not significant). 

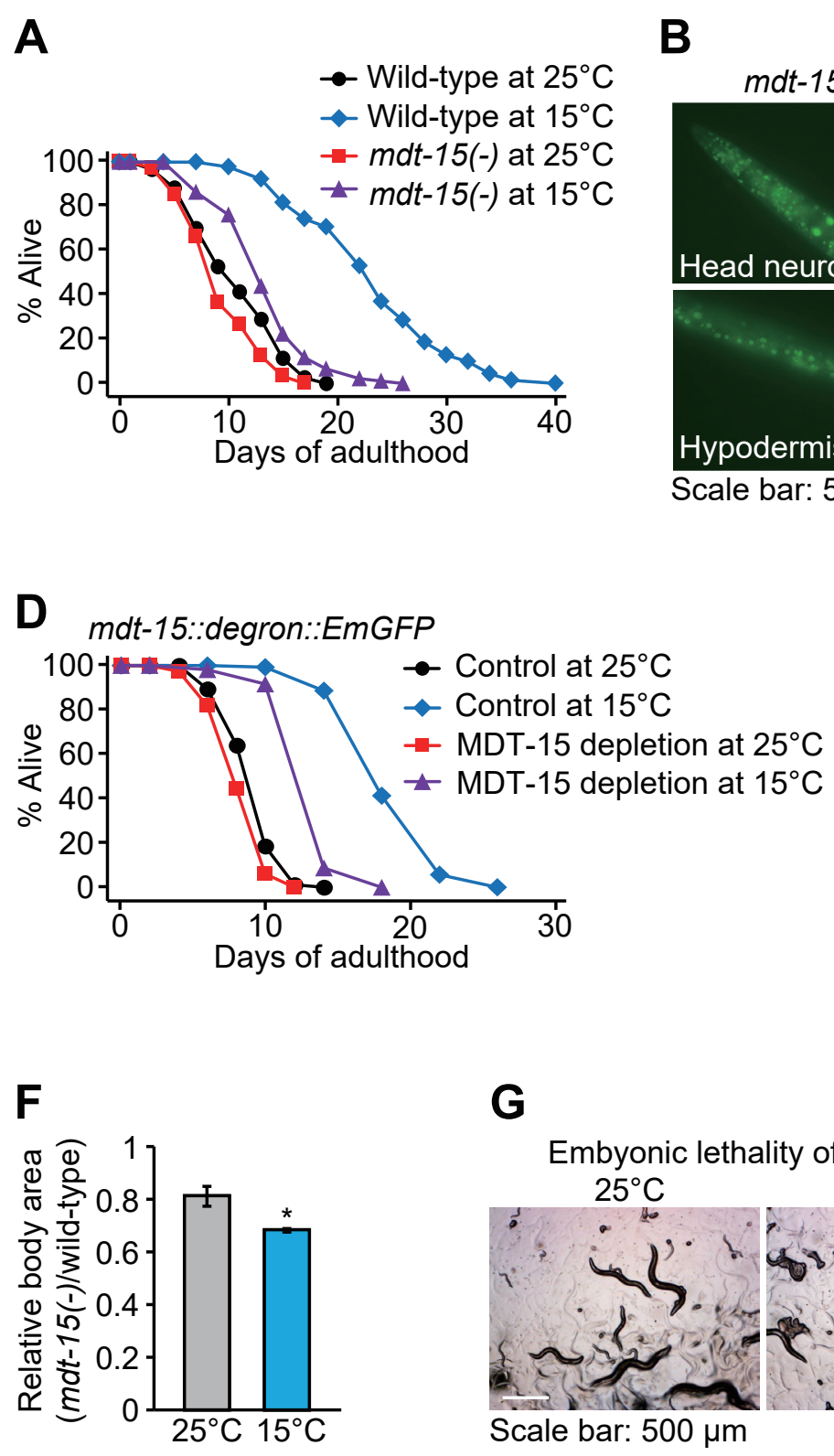

\section{B}

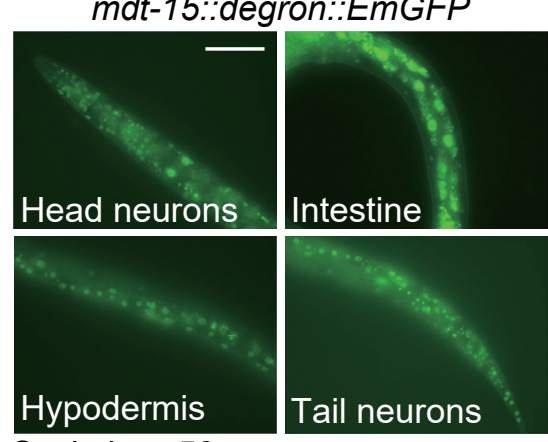

C

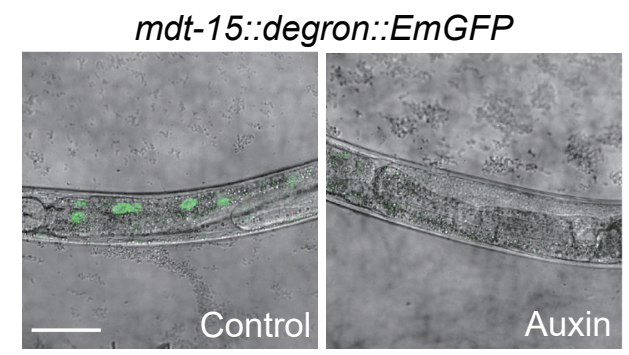

Scale bar: $50 \mu \mathrm{m}$

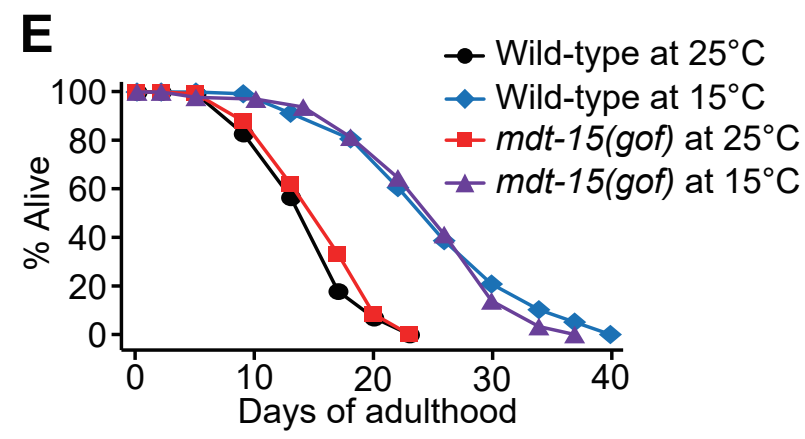

G

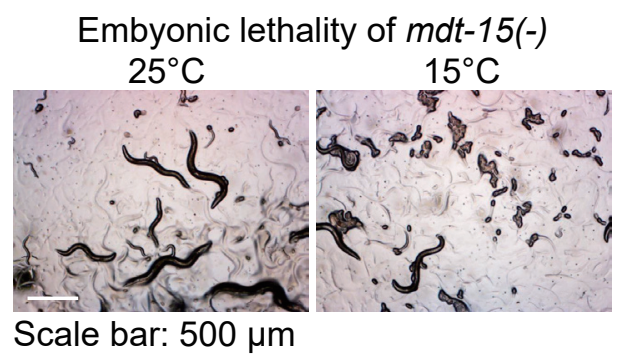

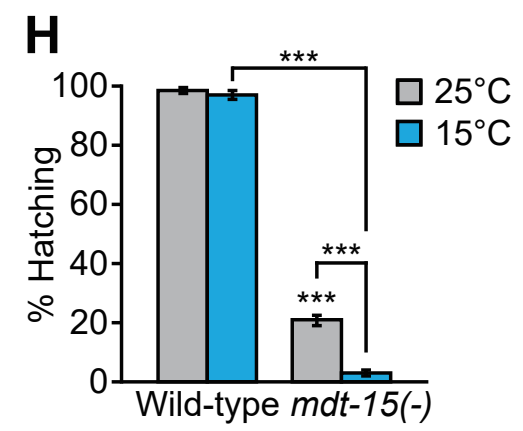


A

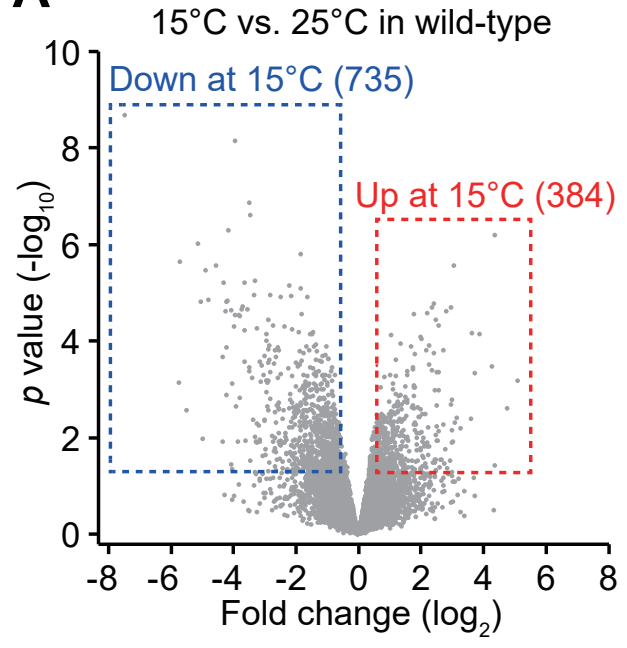

B

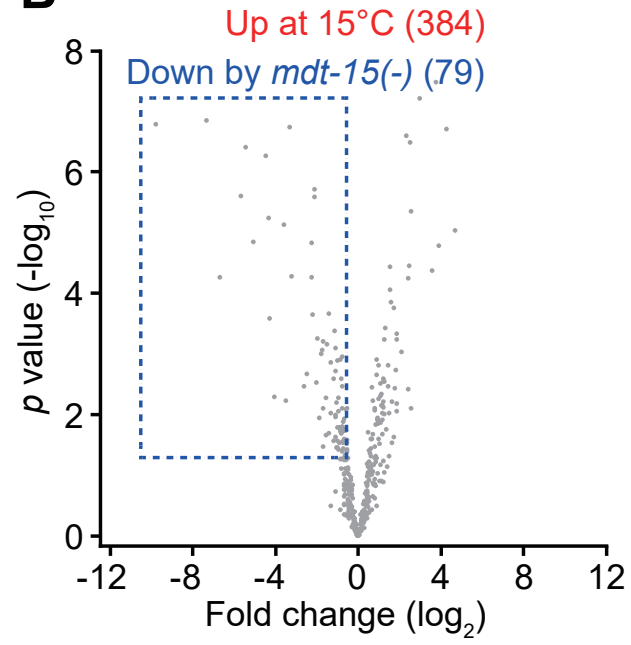

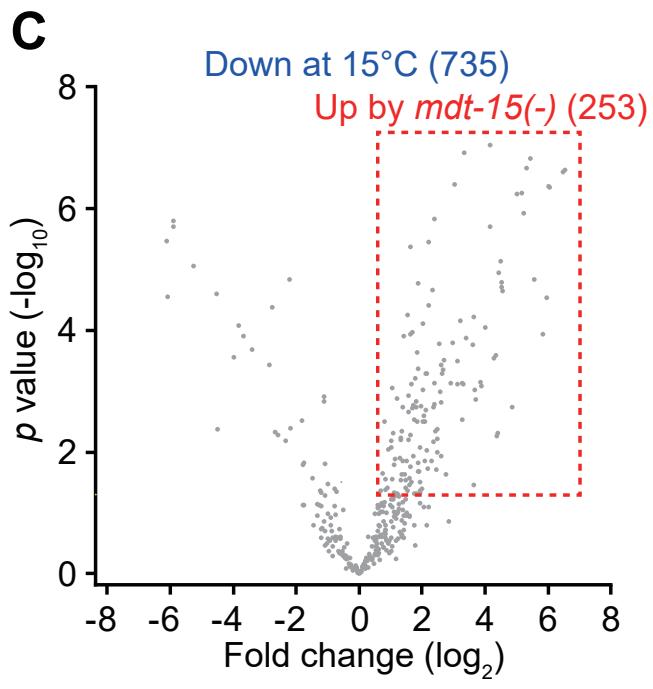

D

MDT-15 dependent up-regulation at $15^{\circ} \mathrm{C}$ Aminoglycan metabolism

Fatty acid metabolism

Organonitrogen compound catabolism Carboxylic acid metabolism Organic acid metabolism Oxidation-reduction process Macromolecular complex assembly Small molecule metabolism Single-organism metabolism

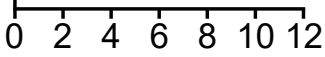

Fold enrichement
E

MDT-15 dependent down-regulation at $15^{\circ} \mathrm{C}$

Chromatin assembly

Aminoglycan catabolic process

Response to heat Immune system process Defense response Response to unfolded protein Response to biotic stimulus

Response to radiation Digestive system development Glycosylation

Carbohydrate metabolic process

Response to abiotic stimulus

Response to stress

Protein complex assembly

Carbohydrate derivative metabolism Macromolecular complex organization 
A

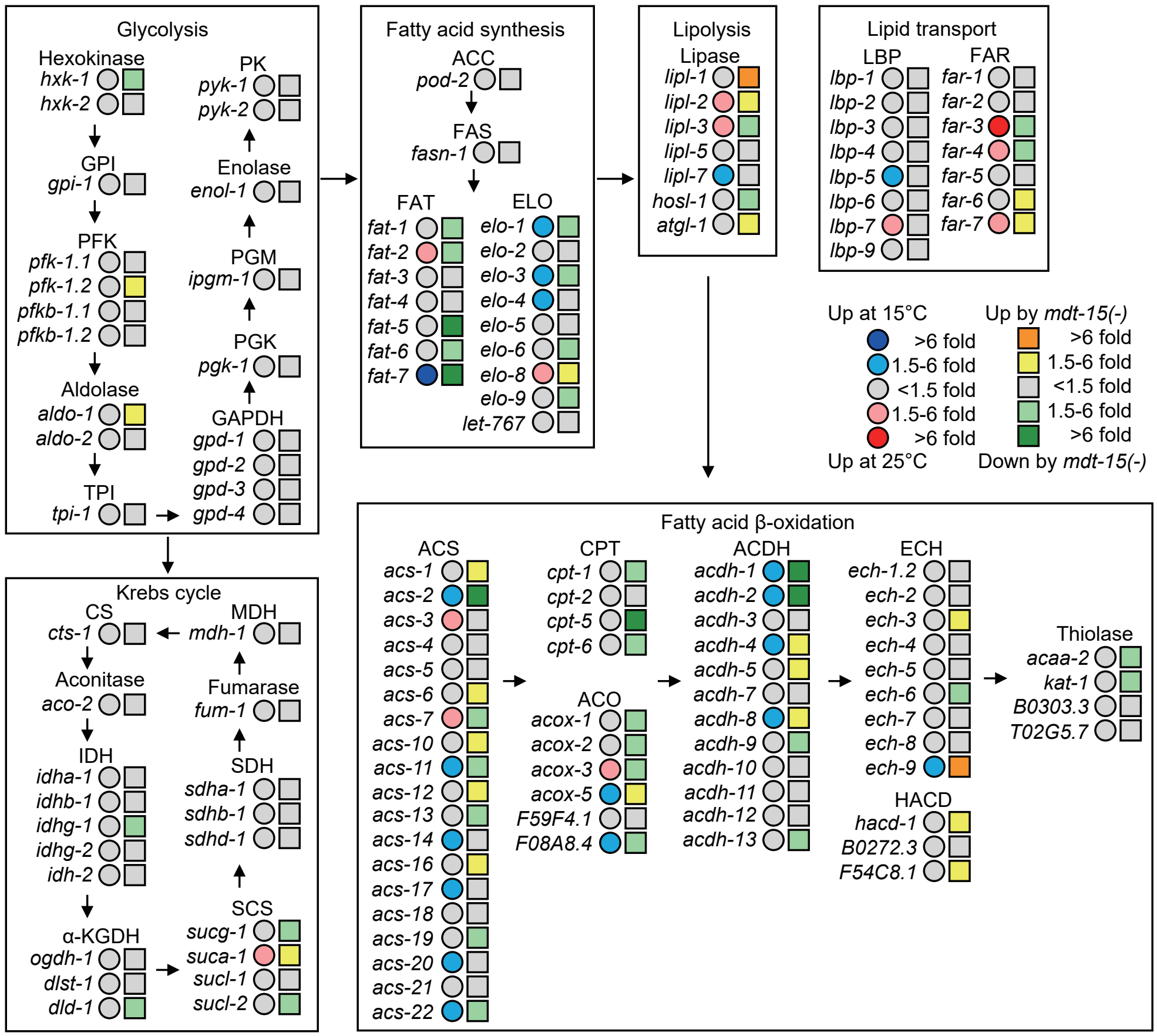

B

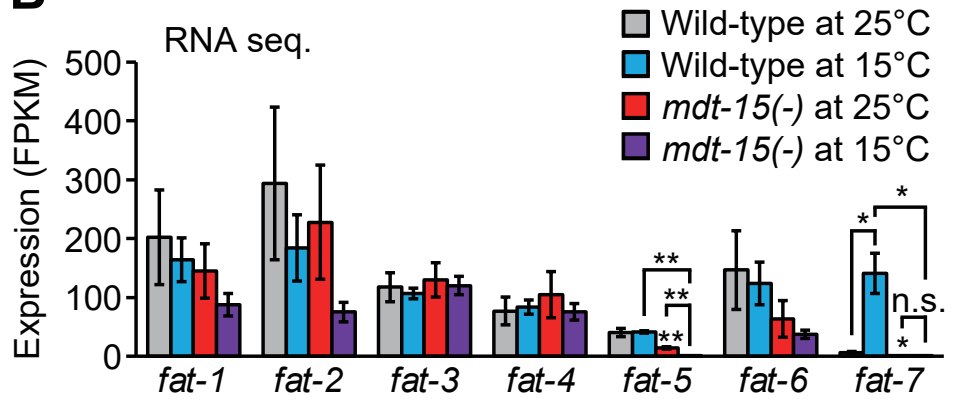

C

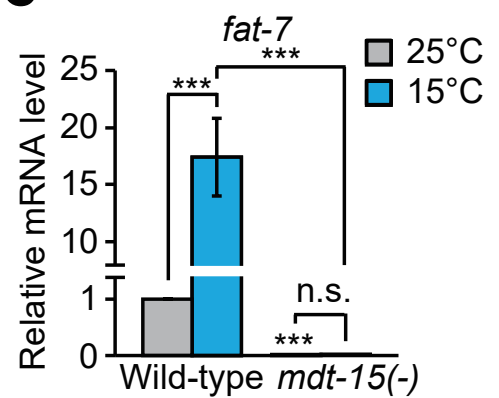

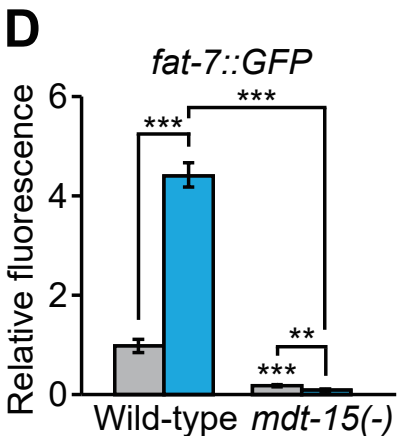


A

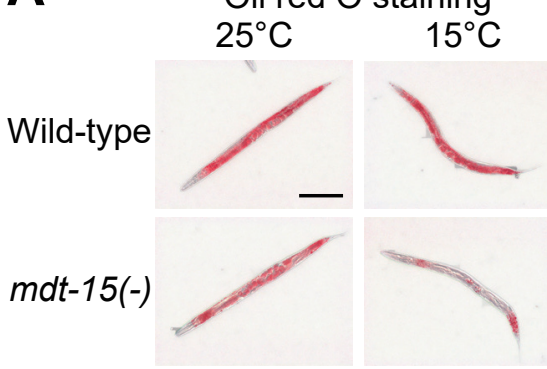

Scale bar: $200 \mu \mathrm{m}$
B

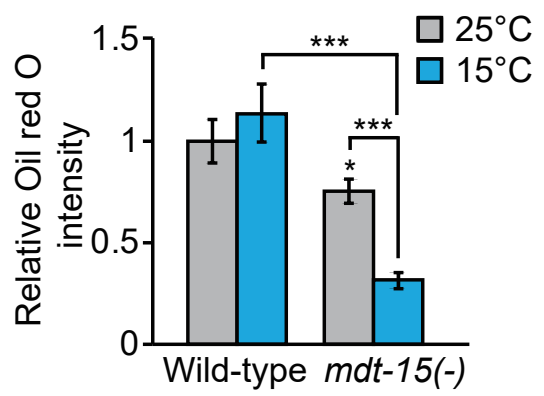

C

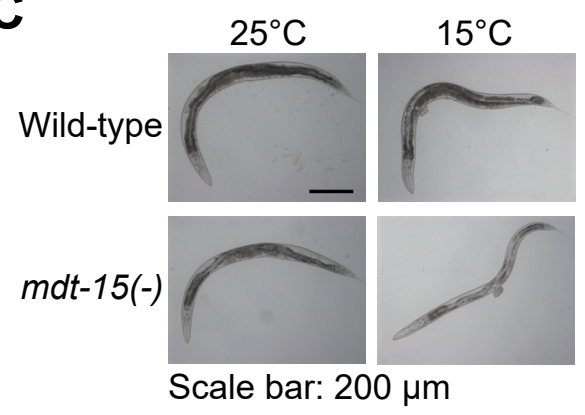

D
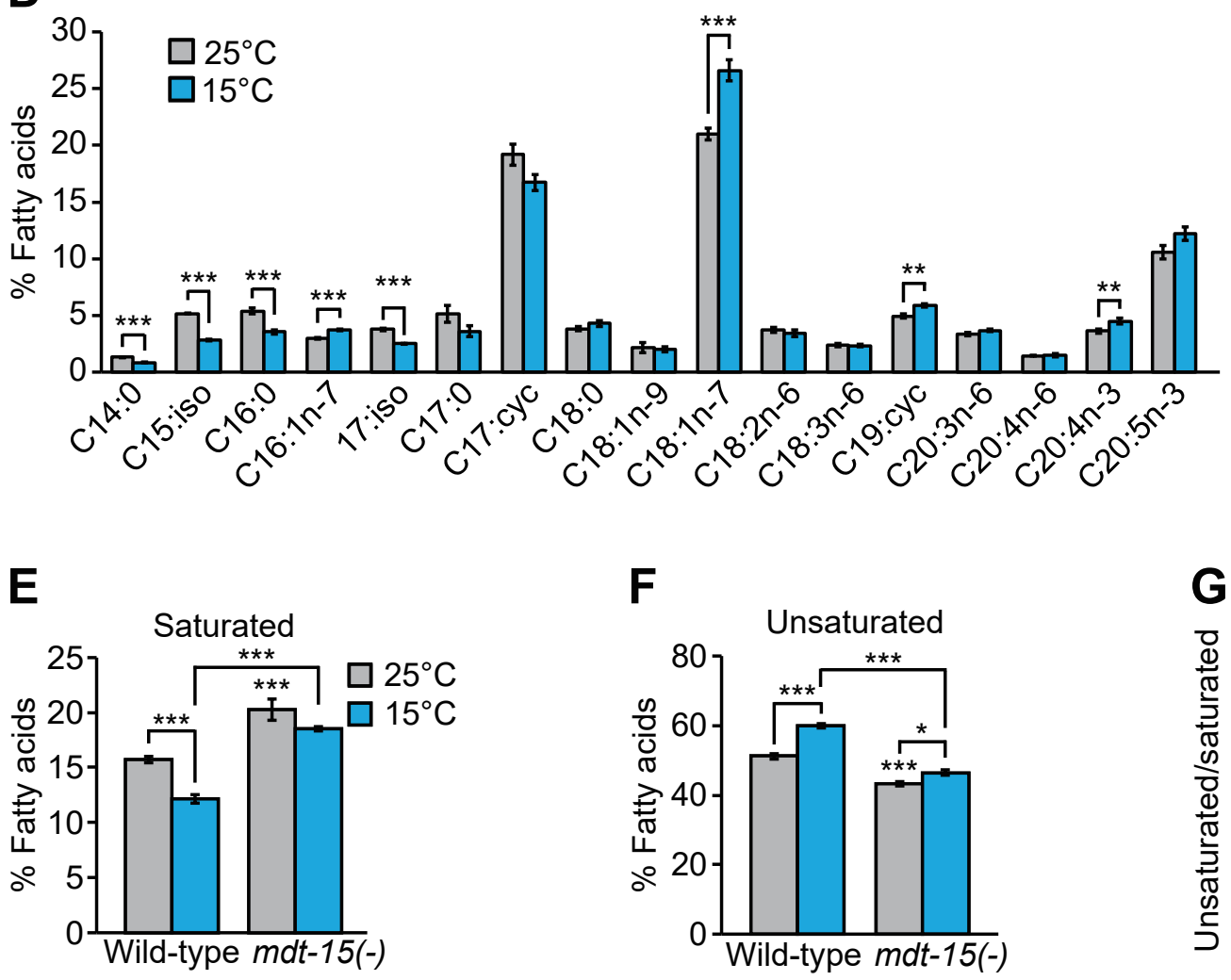

G

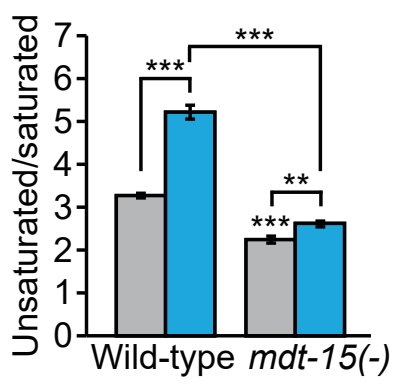



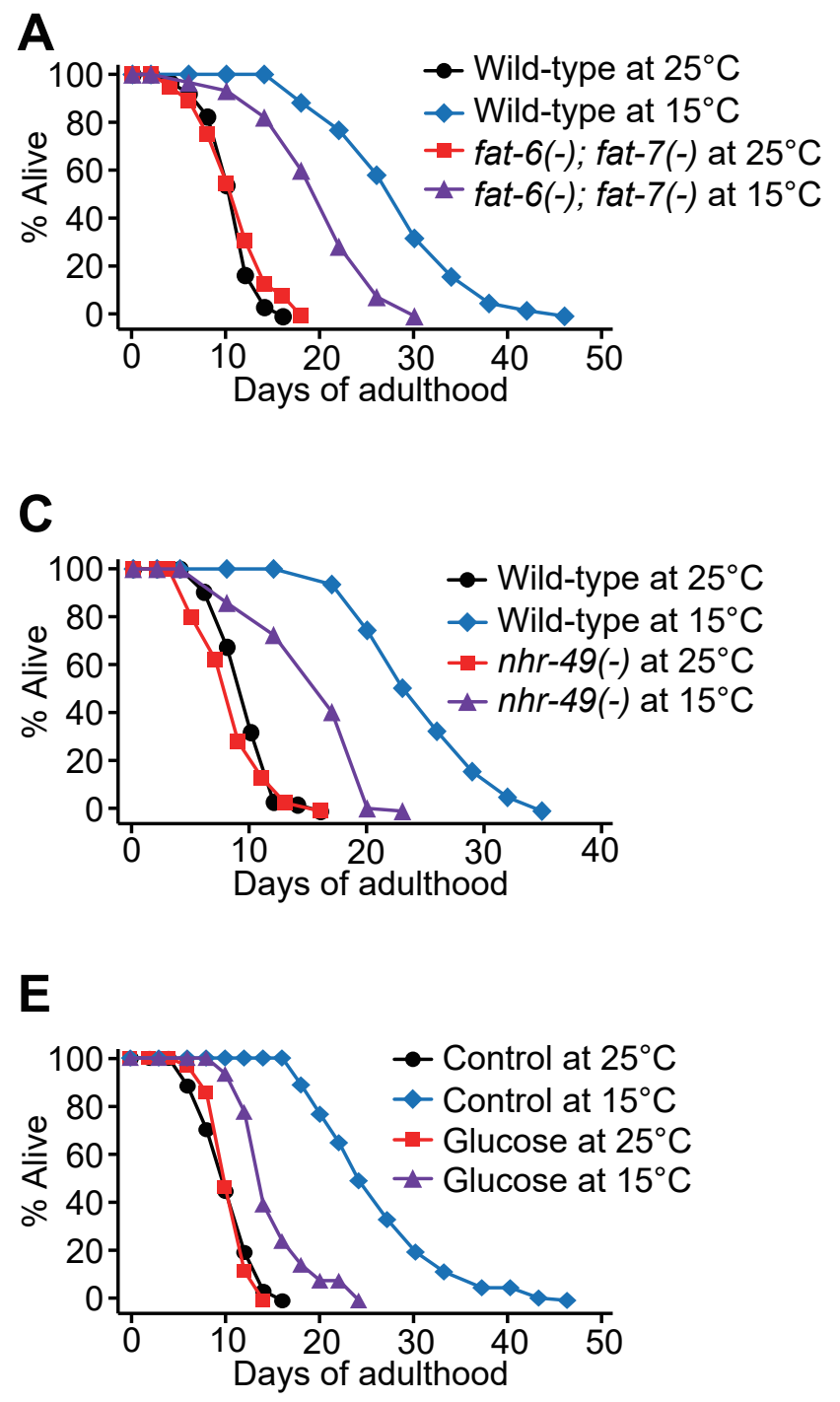
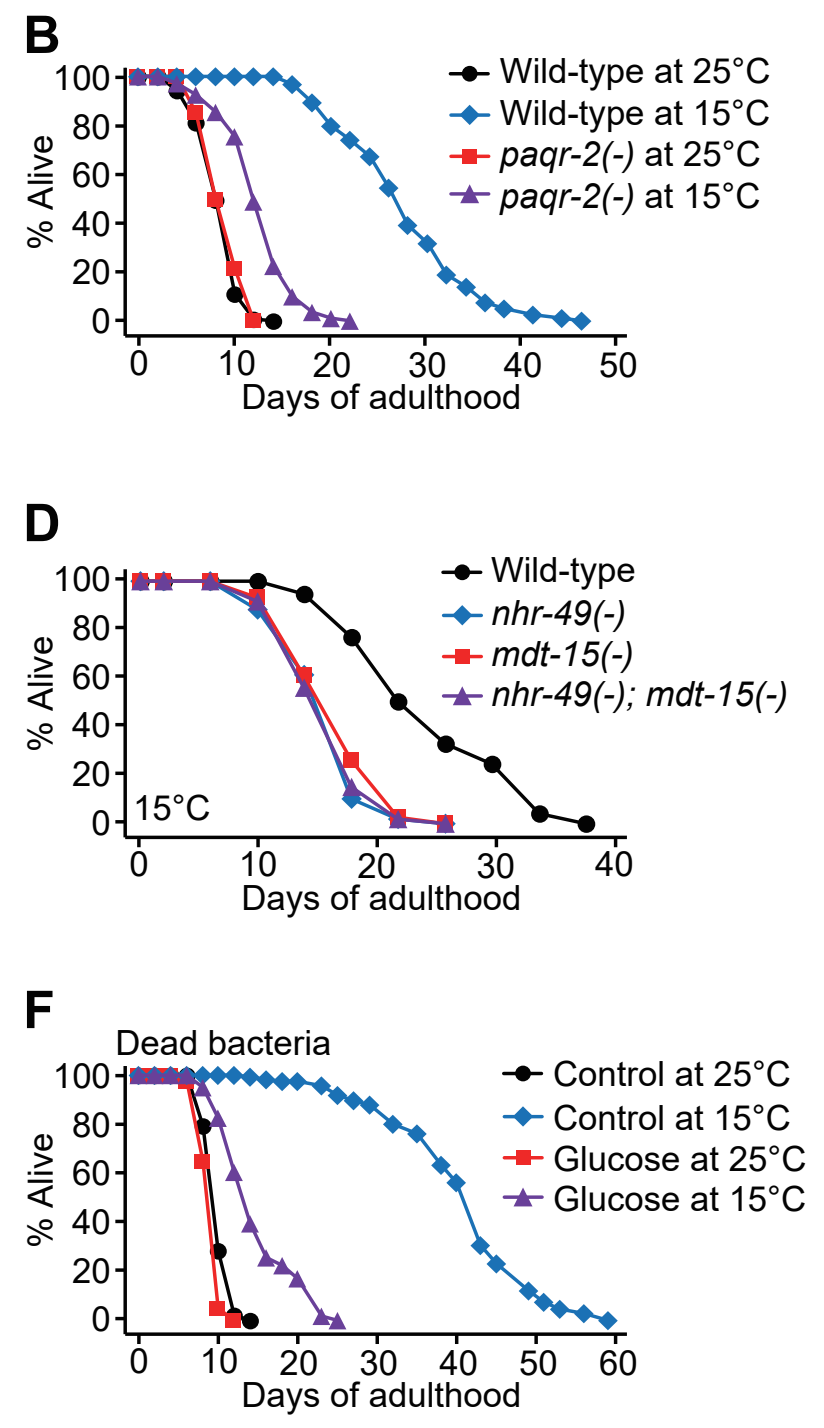


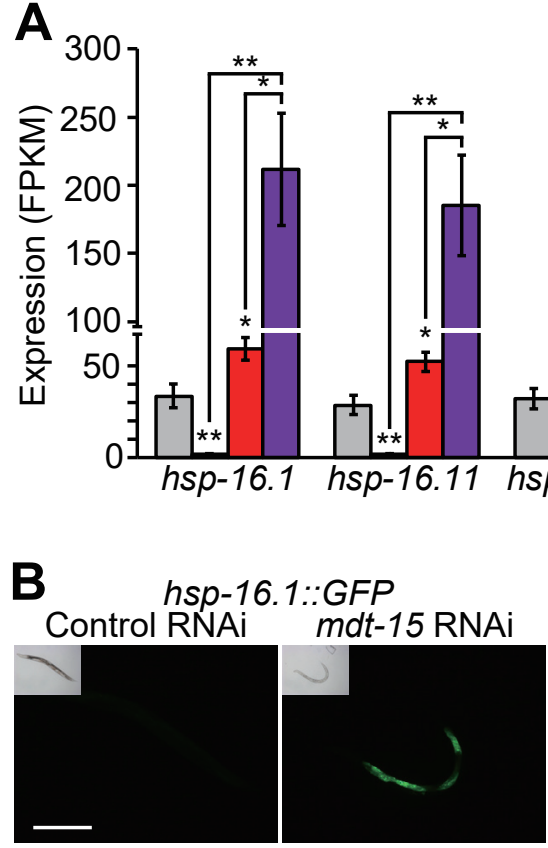

Scale bar: $200 \mu \mathrm{m}$

$\mathbf{E}$
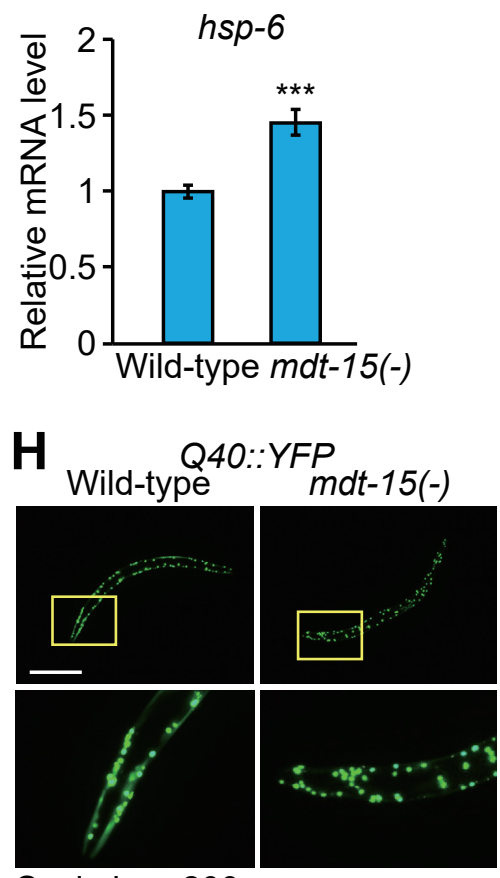

Scale bar: $200 \mu \mathrm{m}$

K

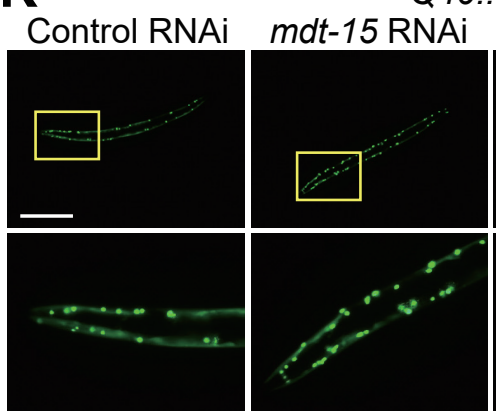

Scale bar: $200 \mu \mathrm{m}$
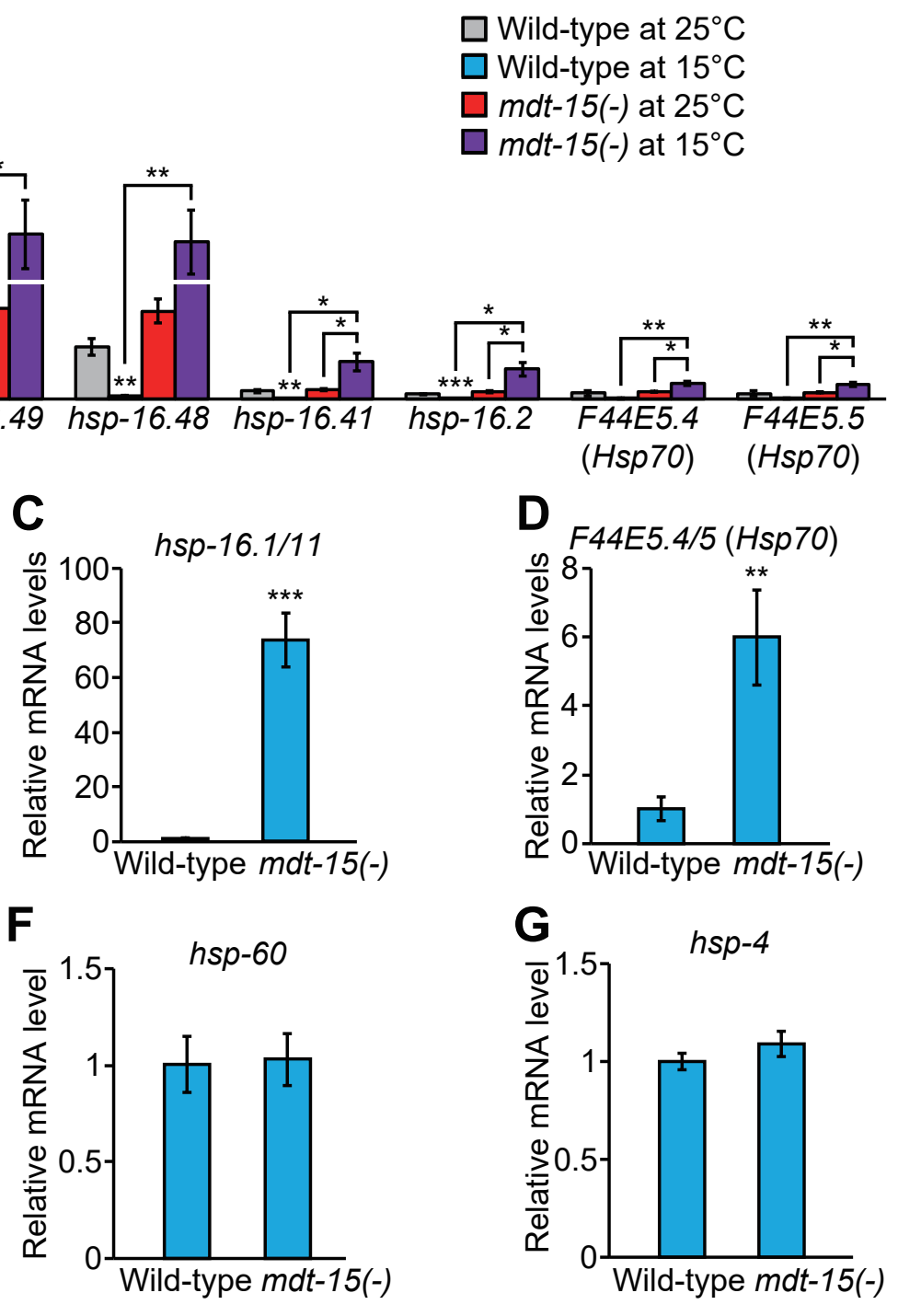

J

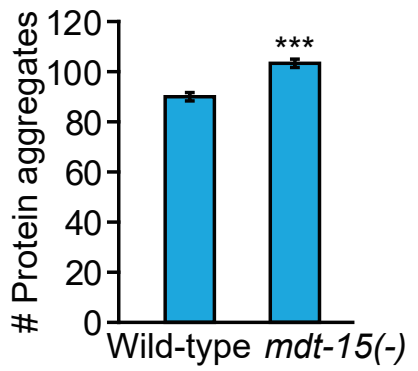

Scale bar: $200 \mu \mathrm{m}$

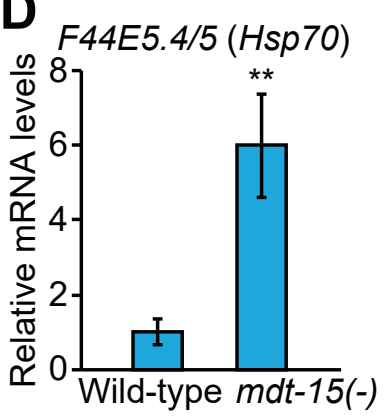

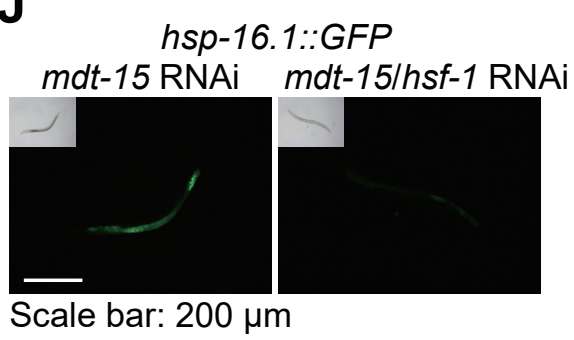

$\mathbf{L}$

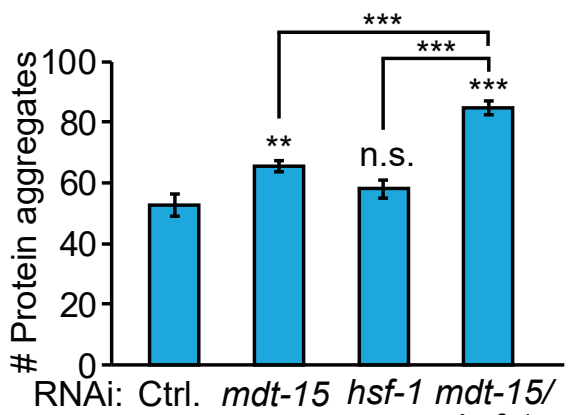

hsf-1 